\title{
Perioperative immunonutrition in hepatectomy: A systematic review and meta-analysis
}

\author{
Chee Siong Wong ${ }^{1,2,3}$, Raaj Praseedom ${ }^{1}$, and Siong-Seng Liau ${ }^{1}$ \\ ${ }^{1}$ Department of Hepatopancreaticobiliary Surgery, Addenbrooke's Hospital, \\ Cambridge University Hospitals NHS Foundation Trust, Cambridge, \\ ${ }^{2}$ University of Edinburgh, ${ }^{3}$ Royal College of Surgeons of Edinburgh, Edinburgh, UK
}

\begin{abstract}
Backgrounds/Aims: The role of immunonutrition (IMN) after liver resections or hepatectomies remains unclear and controversial. We undertook a systematic review to evaluate the effects of IMN on clinical outcomes of patients undergoing hepatectomy. Methods: Main electronic databases were searched for randomised trials reported clinical outcomes or effects of IMN. The systematic review was conducted in accordance with the PRISMA guideline and meta-analysis was analysed using fixed or random-effects models. Results: Eleven RCTs were identified. A total of 1084 patients (529 IMN and 555 Control) were included in the final pooled analysis. Of these patients, 43\% (440/1016) underwent major hepatectomies and the majority are for hepatocellular carcinoma (90\%, 956/1055) with Child-Pugh A disease (89\%, 793/894). IMN significantly reduced post-operative wound infection (risk ratio (RR) $0.65,95 \%$ confidence interval (Cl) 0.43 to $0.96 ; p=0.03$ ). IMN also had a shorter hospital stay (MD -4.97 days, $95 \% \mathrm{Cl}-8.23$ to $-1.72 ; p=0.003$ ). There was no statistically significant in other post-operative morbidities and mortality. Conclusions: Wound infection rate was not significantly different between oral and parenteral IMN group. The length of hospital stay was significantly lower in parenteral IMN group than in oral IMN group. The mortality rates were not affected. Immunonutrition should be recommended routinely as part of the nutritional support in the Enhanced Recovery after Surgery (ERAS) protocol for hepatectomy. (Ann Hepatobiliary Pancreat Surg 2020;24:396-414)
\end{abstract}

Key Words: Immunonutrition; Hepatectomy; Complication; Liver resection

\section{INTRODUCTION}

Perioperative nutritional support (NS) have changed substantially during the last decade with the advent of Enhanced Recovery after Surgery (ERAS). ${ }^{1}$ Perioperative nutritional therapy potentially can maintain hepatocellular function, preserve protein synthesis and attenuate catabolic response to metabolic alteration in liver resection. The primary goal for NS in patients before and after hepatopancreaticobiliary surgery is to quickly restore health and function to patients by minimising catabolic stress response to operation, which can be achieved by (a) Early re-establishment of oral feeding; this is facilitated by the use of ERAS protocols; (b) Support and/or restore normal digestion and intestinal absorption; and (c) Identify nutri- tional deficits preoperatively and to correct them. ${ }^{2}$

Most patients in Western countries undergoing liver resection have no associated cirrhosis and have no need for specialised nutritional support preoperatively and postoperatively. Majority of patients can begin an oral diet safely on the first or second day postoperative day, and they are able to tolerate a full regular diet within 5 days. However, malnourished patients being considered for elective liver resection should receive nutritional supplementation preoperatively and postoperatively by the oral or enteral route. In addition, patients undergoing extensive liver resection, particularly patients with compromised hepatocellular functions (e.g. steatosis, chronic hepatitis, or cirrhosis), may benefit from specialised NS.

The European Society for Parenteral and Enteral Nutri-

Received: April 2, 2020; Revised: May 30, 2020; Accepted: June 7, 2020

Corresponding author: Chee Siong Wong

Department of Hepatopancreaticobiliary Surgery, Addenbrooke's Hospital, Cambridge University Hospitals NHS Foundation Trust, Hills Rd, Cambridge, CB2 0QQ, UK

Tel: +44-7519928415, E-mail: m.wong4@nhs.net

Copyright (C) 2020 by The Korean Association of Hepato-Biliary-Pancreatic Surgery

This is an Open Access article distributed under the terms of the Creative Commons Attribution Non-Commercial License (http://creativecommons.org/ licenses/by-nc/4.0) which permits unrestricted non-commercial use, distribution, and reproduction in any medium, provided the original work is properly cited. Annals of Hepato-Biliary-Pancreatic Surgery • pISSN: 2508-5778 - elSSN: 2508-5859 
tion (ESPEN) guidelines recommend parenteral nutrition in undernourished or malnourished surgical patients, in whom enteral nutrition is not feasible or not tolerated. ${ }^{3}$ The recent American Society for Parenteral and Enteral Nutrition (ASPEN) guidelines recommend postoperative enteral nutrition when feasible within 24 hour and postoperative parental nutrition for patients who cannot meet their energy needs orally or enterally within 5-7 days. ${ }^{4}$ The administration of parenteral nutrition via central vein catheter to well-nourished patients undergoing surgery is associated with a higher incidence of postoperative complications. ${ }^{5}$

The potential benefit of administering immunonutrition (IMN) and antioxidants to patients undergoing HPB surgery is controversial, although of increasing interest. ${ }^{6}$ Short courses of preoperative immune-modulating formulas, using combinations of arginine, $\omega-3$ fatty acids, and other nutrients, have been associated with improved surgical outcomes. These immune-modulating nutrients are key elements of metabolic pathways that promote attenuation of the metabolic response to stress and improve both wound healing and immune function.

Immunonutrition or immune-enhancing diets (IEDs) continue to gain wider use in the care of critically ill and trauma patients. The enteral formulas contain adjunct key nutrients, such as glutamine, arginine, branched-chain amino acid (BCAA), nucleotide, omega-3 fatty acid and beta carotene, ${ }^{7}$ are specially designed to modulate and improve immune function. Most of the randomised controlled trials performed so far in elective surgical patients have evaluated the efficacy of enteral formulas with combination of such immune-modulating substances. Data suggest that these formulas reduce the incidence of infectious and non-infectious complications and shortened hospital stays in various elective surgeries, ${ }^{8}$ however other meta-analysis published conflicted results. ${ }^{9}$

In a larger systematic review and meta-analysis of immunonutrition (including trials of glutamine, arginine, omega-3 fatty acids, RNA, and nucleotides) recently conducted showed that there was no effect on perioperative mortality associated with major abdominal surgery. Compared with control groups, immunonutrition reduced the risk of overall complications (odds ratio [OR] 0.79, 95\% CI, 0.66 to 0.94, 41 trials) and infectious complications (OR 0.58, $95 \%$ CI 0.51 to $0.66,66$ trials), and shortened hospital stay (mean difference -1.79 days, $95 \% \mathrm{CI}-2.39$ to -1.19 , 52 trials). However, trials included in this meta-analysis were rather heterogeneous-mixture of surgical cases including upper gastrointestinal tract, HPB, and colorectal surgery. ${ }^{10}$ Due to inhomogeneity of various previous metaanalyses, the role for IMN (e.g. enteral or parenteral supplementation with arginine, glutamine, nonessential fatty acids, branched chain fatty acids, nucleotides, or RNA), remains unclear.

Minor hepatectomy is defined as the removal of two or less segments of the liver, which is often described as lobectomy. Some author considers that major hepatectomy involves removal of four or more segments of the liver. ${ }^{11}$ Most surgical textbooks defined major hepatectomy as resection of three or more contiguous liver segments. ${ }^{12,13}$ In this review, we follow the latter definition. Major hepatectomy is indicated for a large tumour $(>5 \mathrm{~cm})$ that involves most of the right or left liver or a small tumour if it is close to the liver hilum, encroaching or involving the right or left portal pedicle. ${ }^{12}$ Regardless of the approach used for resection, tumours-free resection margins should be achieved, not only for the primary hepatic malignancies, but also for liver metastases. Generally, achieving adequate margins (approximately $1 \mathrm{~cm}$ of surrounding liver tissue) is desirable, although the prognostic significant of surgical margins for patients who received preoperative chemotherapy for colorectal cancer liver metastases (CRLM) is a matter of debate. ${ }^{13}$ Hepatectomy, as a rule, should only be performed in Child-Pugh A cirrhotic patients. Child-Pugh $\mathrm{B}$ or $\mathrm{C}$ patients are at a prohibitive risk of early liver failure even after a minor hepatectomy or mere laparotomy. ${ }^{14}$

The use of immune-enhancing formulas enriched with glutamine, omega-3 polysaturated fatty acids ( $\omega-3$ FAs), arginine and ribonucleic acids (RNA) has been found to improve clinical outcomes. A number of previous metaanalyses have evaluated the efficacy of immune-enhancing formulas and have shown superior outcomes compared with standard formulations in certain patient populations. $^{8,15-17}$ To date, there is no data to support the routine use of nutritional support in well-nourished and malnourished patients undergoing liver resection or hepatectomy. Our aim of this present meta-analysis was to examine the effects of perioperative immunonutrition support in patients undergoing hepatectomy. 


\section{MATERIALS AND METHODS}

\section{Search strategy}

The systematic review was conducted in accordance with the Preferred Reporting Items for Systematic Reviews and Meta-analyses (PRISMA) guideline. ${ }^{18}$ This review was registered with the International Prospective Register of Systematic Review (PROSPERO) (Registration No. CRD42017073787) and has been reported in line with AMSTAR (Assessing the methodological quality of systematic reviews) Guidelines. Main electronic databases MEDLINE via PubMed (1966 to current), EMBASE (via OVID) (1980 to current), Scopus (1995 to current), Web of Science (ISI Web of Knowledge) (1900 to current), Cochrane Central Register of Controlled Trials (CENTRAL) and the Cochrane Library, and clinical trial registries (ClinicalTrial.gov and WHO International Clinical Trials Registry Platform (ICTRP)) were searched for studies reporting outcomes of immunonutrition in hepatectomy using following search terms or key words. Key words'immunonutrition'; 'enteral'; 'hepatobiliary surgery', 'hepatopancreaticobiliary surgery' or 'liver surgery'; 'glutamine'; 'omega-3'; 'fish oil'; 'fatty acid'; 'arginine'; 'hepatectomy'; 'liver resection'; 'nutrition'; 'complication'.

These terms were searched using Boolean operators (AND and OR) and appropriate Medical Subject Headings
(MeSH) terms were combined in the search builder. A truncation symbol (e.g. adding superscript asterisk * at the end of a word) was used wherever appropriate. Searches were not restricted by publication year, publication status or language. Relevant conference abstracts and proceedings presented at the American Surgical Association, Association of Surgeons of Great Britain and Ireland, Annual Conference of American College of Surgeons were hand-searched. A manual search was also conducted for reference lists in the included studies.

\section{Study selection and data extraction}

Eligible studies were included provided they met the following inclusion criteria:

(1) randomised controlled trial (RCT);

(2) patient undergoing hepatectomy (either open or laparoscopic approach; anatomical or non-anatomical resection; for benign and malignant liver tumors);

(3) with reported outcomes comparing immunonutrition and control with or without standard nutritional supplementation.

We excluded following studies: non-RCT (case series, case-control study, and cohort study), narrative or expert reviews, and animal studies or trials. Article reference lists were scrutinised for relevant articles independently by two authors (CSW and SSL). Any differences in opin-

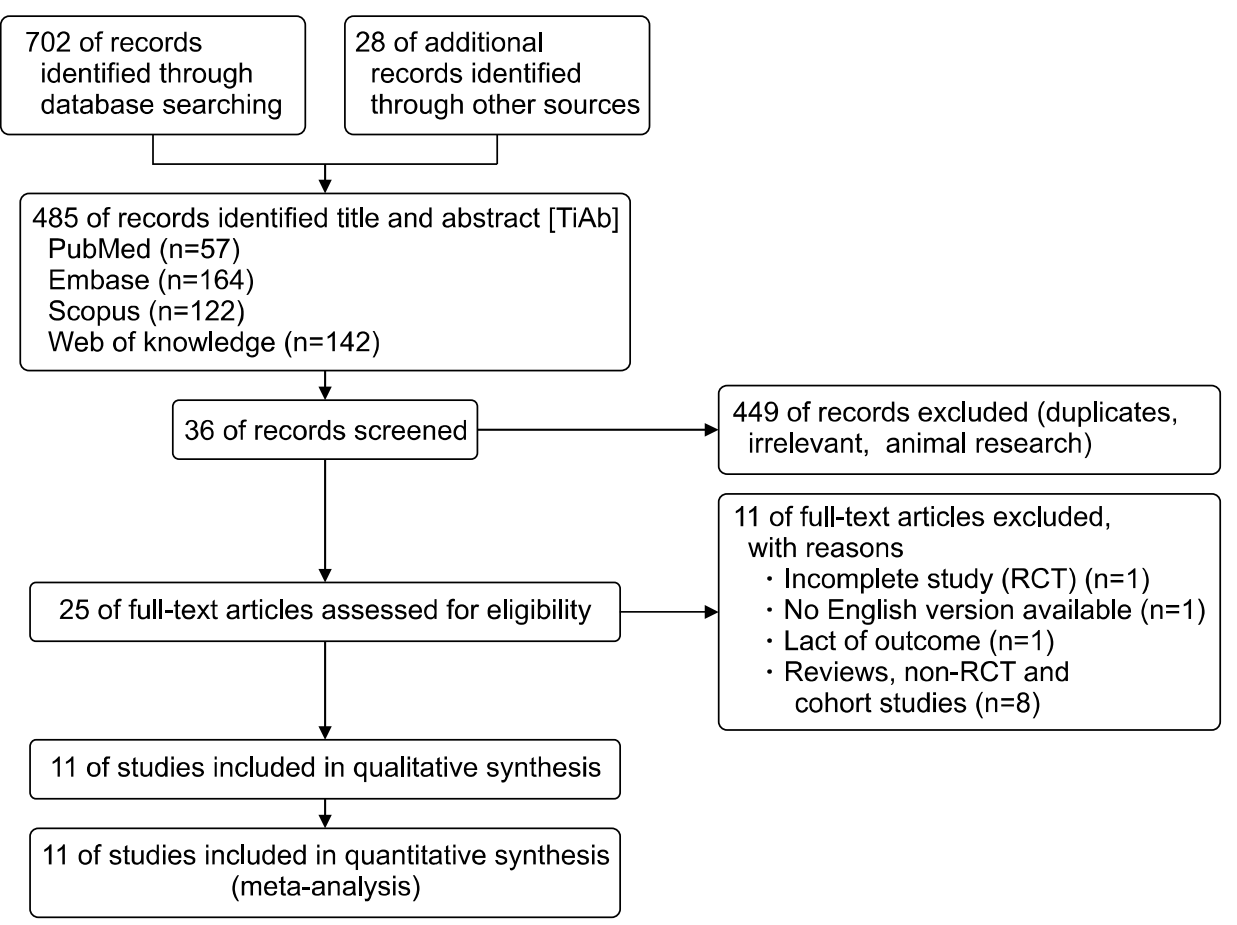

Fig. 1. PRISMA flow diagram. RCT, randomised controlled trial. 
ion were resolved through discussion or by a third reviewer (RP).

Data available from the study for each outcome of interest were extracted and recorded in a formulated contingency table using Microsoft Excel spread sheet. Categorical nominal data (dichotomous or binary data) were reported in a $2 \times 2$ table by quantifying the presence or absence of that outcome of interest. Continuous data, such as length of hospital stay (days), were represented by mean and standard deviation (mean \pm S.D.). If the mean and standard deviation (variance) of continuous data were not available, the median, range and sample size will be used to estimate the mean and variance using the formula proposed by Hozo et al. ${ }^{19}$

The Jadad scale was used to evaluate the methodological quality of the published randomised control trial. ${ }^{20}$ Three main items were scored (randomisation, double blinding, and withdrawals and dropouts). Scores range from 0 (poorest) to 5 (best). Bias risk was evaluated as per Cochrane methodology. ${ }^{21}$ Potential sources of bias are identified (selection bias, performance bias, detection bias, attrition bias, reporting bias) and graded as 'low risk of bias', 'high risk of bias', or 'risk of bias unclear'. Publication bias is visually assessed by using funnel plots. Asymmetrical funnel plots may indicate publication bias, or be due to exaggeration of treatment effects in small studies of low quality. ${ }^{22}$

\section{Statistical analysis}

A meta-analysis was performed using the software package Review Manager (RevMan) Version 5.3.5 (Java 664 bits) to characterise the effect of various dichoto- mous and continuous outcomes. Subgroup analysis was performed by using effect modification crude analysis technique to stratify and measure the 'true' effect of intervention on the outcome of interest. Sensitivity analysis was also performed to eliminate certain degree of bias in the pooled result of meta-analysis. Outcomes of dichotomous (binary) and continuous variables were measured using Mantel-Haenszel fixed-effect ${ }^{23}$ and DerSimonian and Laird random-effects model. ${ }^{24}$ The degree of heterogeneity observed in the result was quantified using the I-squared $\left(\mathrm{I}^{2}\right)$, which is presented as a percentage. A significant heterogeneity presents when $\mathrm{I}^{2}>50 \%{ }^{25}$ Random effects model were used in reporting all the results unless stated otherwise.

\section{RESULTS}

The systematic review identified 485 citations, of which eleven RCSTs $^{26-36}$ were identified, a total of 1190 patients recruited and included 1084 patients randomised in the final pooled analysis. A PRISMA flow diagram of the search strategy is presented in Fig. 1. Of these patient 529 received immunonutrition supplementation and $555 \mathrm{did}$ not (840 men and 244 women) (Fig. 2A). All eligible studies were published between 1994 and 2017. The largest trial was conducted by Zhang et al. ${ }^{36}$

Standard criteria for inclusion and exclusion criteria were defined in all included studies; however, there were differences between protocols of each study. Some trials reported both major and minor liver resection, ${ }^{26-30,32,36}$ only major resection, ${ }^{34}$ and the rest liver resection (unspecified). ${ }^{31,33,35,36}$ A total of 440 patients and 576 patients underwent major and minor hepatic resection respectively-of
A

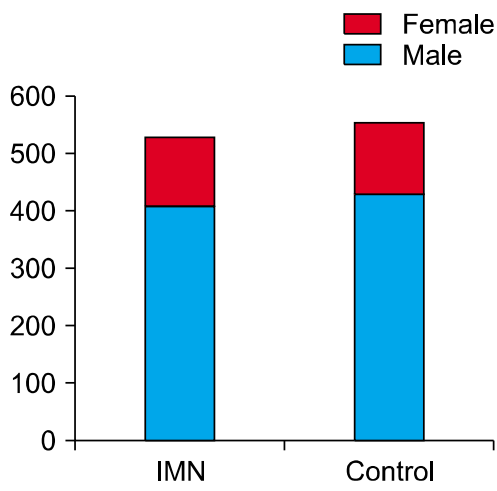

B

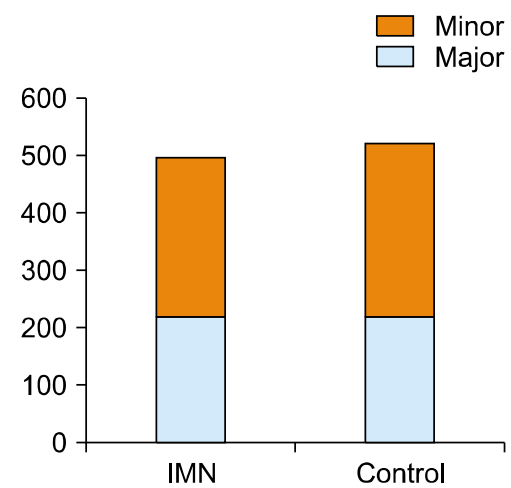

C

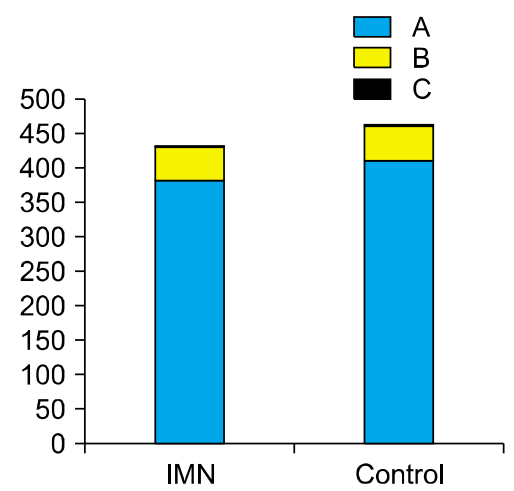

Fig. 2. (A) Histogram depicts the proportion or number of male and female patients in both IMN and control group. (B) Histogram depicts the number of major and minor liver resection in both IMN and control group. (C) Histogram depicts the proportion on Child-Pugh A, B, and $\mathrm{C}$ in both IMN and control group. IMN, immunonutrition. 
which 496 patients were assigned to IMN group compared to 520 patients in control (Fig. 2B). There were eight studies $^{27-29,31-33,35,36}$ reported and stratified patients' prognosis of chronic liver disease according to Child-Pugh score. Of that there were 382 patients with Child-Pugh A in IMN group compared to 411 in the control group (Fig. 2C).

Enteral IMN was administered by oral (and/or nasogastric) route in seven studies ${ }^{27-30,33-35}$ and by parenteral route in four studies (Table 1). 26,31,32,36 A summary of randomised controlled trials comparing the characteristics and outcomes of immunonutrition versus controlled group was summarised in Table 2 .

\section{Quality assessment}

The quality of all clinical trials was evaluated using the Jadad score. Studies with 3 or more of a maximum of 5 points are accounted as high quality. Mode (1 and 3), median (3), and range (1-5) as calculated in the bracket.
A summary of Jadad scoring is as below (Table 3).

The methodological quality of each included study was assessed using the quality check list supplied in the Cochrane Handbook for Systematic Reviews of Interventions, which contains six items: random sequence generation, allocation concealment, blinding of participants and personnel, blinding of outcome assessment, incomplete outcome data and selective reporting.

There were eleven studies included for risk of bias assessment. Individual sources of bias in studies are described below. Figs. 3 and 4 provide a graph and a table summary respectively for risk of bias in all included studies.

\section{Randomisation}

All studies except one ${ }^{29}$ described the methods of patient randomisation. Most of the trials had adequate generation of allocation sequence either by a random number table (Kikuchi et al. $^{35}$ ), a computer random number gen-

Table 1. Characteristic of the RCT studies included in this meta-analysis

\begin{tabular}{|c|c|c|c|c|c|}
\hline Author (year) & Country & $\begin{array}{l}\text { Timing of } \\
\text { intervention }\end{array}$ & $\begin{array}{l}\text { Duration of } \\
\text { intervention } \\
\quad \text { (days) }\end{array}$ & Type of immunonutrition & $\begin{array}{l}\text { Route of } \\
\text { nutrition }\end{array}$ \\
\hline Fan et al. $(1994)^{26}$ & Hong Kong & Pre-op (7 days) & 7 & $\begin{array}{l}\text { BCAAs, } 1.5 \mathrm{~g} / \mathrm{kg} / \text { day } \\
\text { (maximum of } 1.75 \text { liters per day) }\end{array}$ & $\mathrm{PN}$ via $\mathrm{CVC}$ \\
\hline $\begin{array}{l}\text { San-In Group of } \\
\text { Liver Surgery }(1997)^{27}\end{array}$ & China & $\begin{array}{l}\text { Post-operation } \\
\text { (2-3 weeks) }\end{array}$ & 365 & $\begin{array}{l}\text { BCAAs (Aminoleban EN, } \\
\text { Otsuka Pharmaceutical company, Tokyo) } \\
-50 \text { g BD }\end{array}$ & Oral \\
\hline Meng et al. $(1999)^{28}$ & Hong Kong & $\begin{array}{l}\text { Post-op day } \\
\text { (as oral intake } \\
\text { resume) }\end{array}$ & 84 & $\begin{array}{l}\text { BCAAs (Aminoleban EN, } \\
\text { Otsuka Pharmaceutical company, Tokyo) } \\
-50 \text { g TDS }\end{array}$ & Oral \\
\hline Okabayashi et al. $(2008)^{29}$ & Japan & Pre-op ( 2 weeks) & - & $\begin{array}{l}\text { BCAAs (Aminoleban EN, } \\
\text { Otsuka Pharmaceutical company, Tokyo) } \\
-100 \text { g OD }\end{array}$ & Oral \\
\hline Mikagi et al. $(2011)^{30}$ & Japan & Pre-op (5 days) & 5 & $\begin{array}{l}\text { Omega-3 fatty acids, arginine, nucleotides } \\
\text { (IMPACT, Ajinomoto Pharma, Tokyo) }\end{array}$ & Oral \\
\hline $\mathrm{Wu}$ et al. $(2012)^{31}$ & China & Post-operation & 5 & $\begin{array}{l}\text { Omega-3 fatty acids } \\
\text { (Omegaven, Fresenius-Kabi) }\end{array}$ & $\mathrm{PN}$ via $\mathrm{CVC}$ \\
\hline Gong et al. $(2016)^{32}$ & China & Post-operation & 5 & $\begin{array}{l}\text { Omega-3 fatty acids } \\
\text { (Omegaven, Sino-Swed, JiangSu) }\end{array}$ & $\mathrm{PN}$ via $\mathrm{CVC}$ \\
\hline Seguin et al. $(2016)^{33}$ & France & Pre-op (7 days) & 10 & $\begin{array}{l}\text { Omega-3 fatty acids, arginine, RNA } \\
\text { (IMPACT, Novartis, Bern) }\end{array}$ & $\begin{array}{l}\text { Oral \& } \\
\text { nasogastric } \\
\text { tube }\end{array}$ \\
\hline Uno et al. $(2016)^{34}$ & Japan & Pre-op (5 days) & - & $\begin{array}{l}\text { Arginine, EPA and nucleotides } \\
\text { (IMPACT, Nestle, Kobe) }\end{array}$ & Oral \\
\hline Kikuchi et al. $(2016)^{35}$ & Japan & Pre-op (30 days) & 365 & $\begin{array}{l}\text { BCAAs (Livact Granules, Ajinomoto, } \\
\text { Tokyo)-4.74 g TDS }\end{array}$ & Oral \\
\hline Zhang et al. $(2017)^{36}$ & China & $\begin{array}{l}\text { Post-operation } \\
\text { (post-op day 1) }\end{array}$ & 5 & $\begin{array}{l}\text { Omega- } 3 \text { fatty acids } \\
\text { (Omegaven, Fresenius- Kabi, Germany) }\end{array}$ & $\mathrm{PN}$ via $\mathrm{CVC}$ \\
\hline
\end{tabular}

PN, parenteral nutrition; CVC, central venous catheter; BCAAs, branched chain amino acids; EPA, eicosapentaenoic acid; OD, once a day; $\mathrm{BD}$, twice a day; TDS, Three times a day 


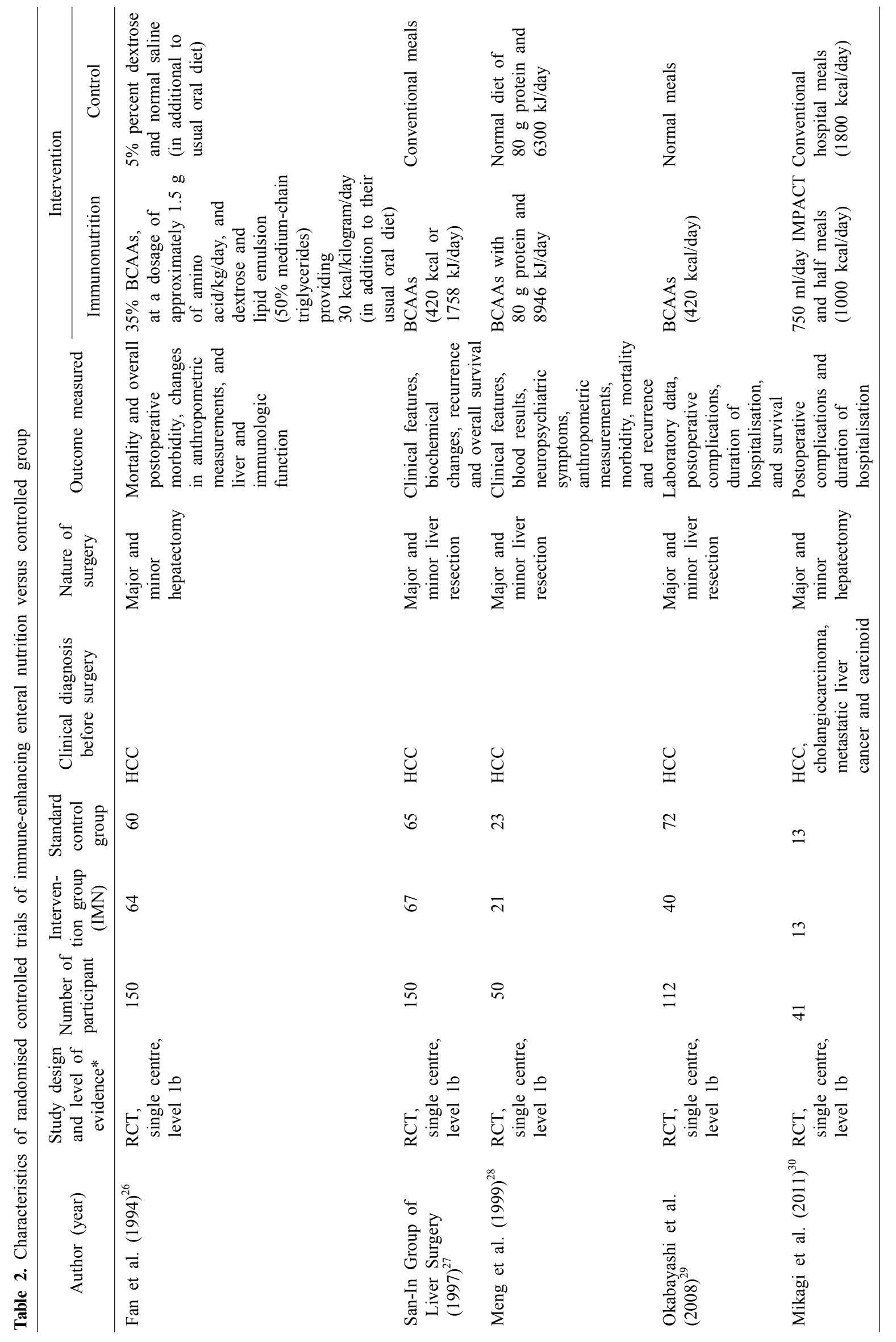




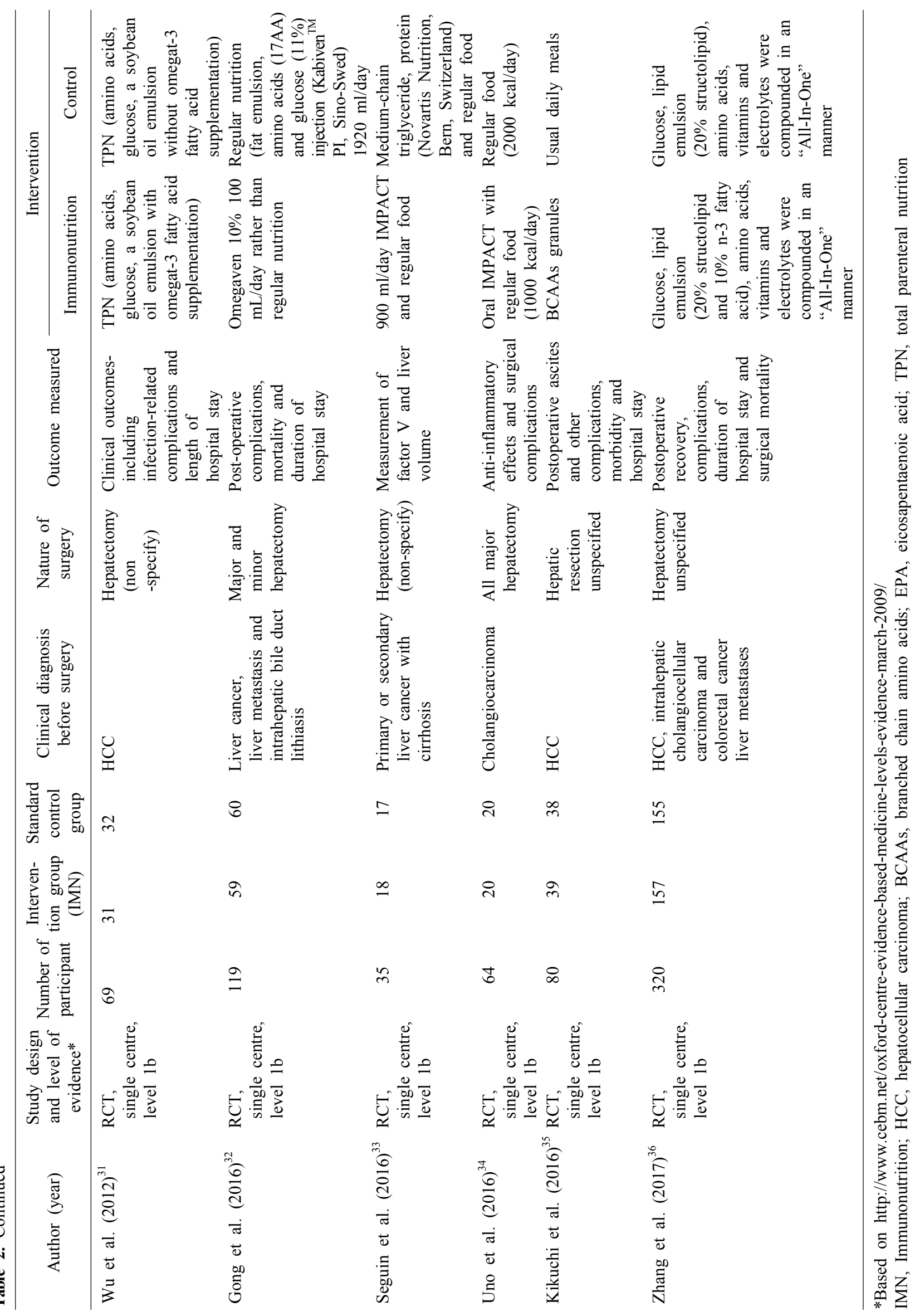




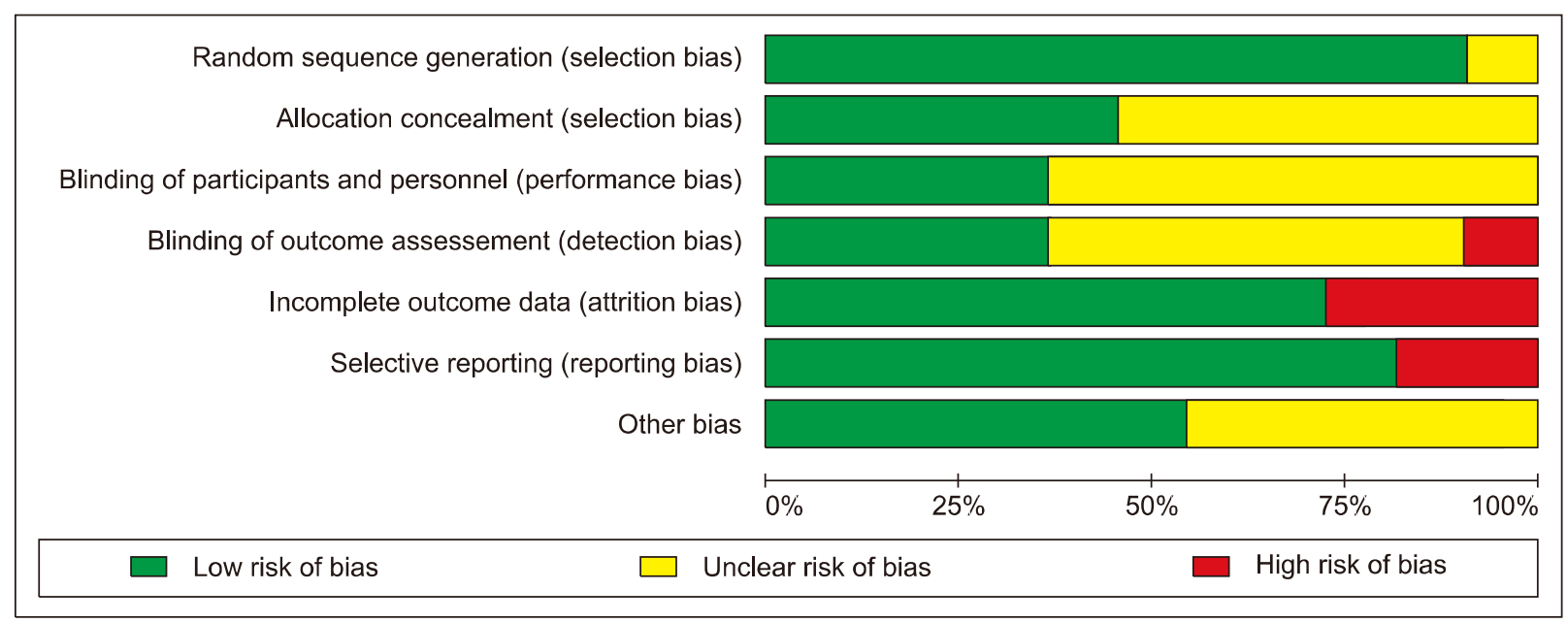

Fig. 3. Risk of bias graph: review authors' judgements about each risk of bias item presented as percentages across all included studies.

Table 3. Jadad scoring

\begin{tabular}{lcccc}
\hline \multicolumn{1}{c}{ Author (year) } & $\begin{array}{c}\text { Randomi- } \\
\text { sation } \\
\end{array}$ & $\begin{array}{c}\text { Blinding } \\
\text { (R) }\end{array}$ & $\begin{array}{c}\text { Dropout } \\
\text { (B) }\end{array}$ & Total \\
\hline Fan et al. (1994) & 1 & 0 & 0 & 1 \\
San-In Group of & 1 & 0 & 0 & 1 \\
Liver Surgery (1997) & & & & \\
Meng et al. (1999) & 2 & 1 & 0 & 3 \\
Okabayashi et al. $(2008)^{29}$ & 1 & 0 & 0 & 1 \\
Mikagi et al. $(2011)^{30}$ & 1 & 0 & 1 & 2 \\
Wu et al. $(2012)^{31}$ & 1 & 2 & 0 & 3 \\
Gong et al. $(2016)^{32}$ & 1 & 1 & 0 & 2 \\
Seguin et al. $(2016)^{33}$ & 2 & 2 & 1 & 5 \\
Uno et al. $(2016)^{34}$ & 2 & 0 & 1 & 3 \\
Kikuchi et al. $(2016)^{35}$ & 2 & 1 & 1 & 4 \\
Zhang et al. $(2017)^{36}$ & 2 & 2 & 1 & 5 \\
\hline
\end{tabular}

erator (Meng et al.; ${ }^{28}$ Uno et $\mathrm{al}^{34}$ ) or a block randomisation (Wu et al.; ${ }^{31}$ Seguin et al.; ${ }^{33}$ Zhang et al. ${ }^{36}$ ). Five included trials described the used of sealed envelopes in the generation of allocation concealment (Meng et al.; ${ }^{28}$ Seguin et al.; ${ }^{33}$ Uno et al.; ${ }^{34}$ Kikuchi et al.; ${ }^{35}$ Zhang et $\mathrm{al}^{36}$ ). The remaining of the authors did not describe methods of allocation concealment.

\section{Blinding}

Two studies described blinding of participants and personnel (Wu et al.; ${ }^{31}$ Gong et al. ${ }^{32}$ ). Three studies described blinding an assessor of outcomes (Meng et al. ${ }^{28} \mathrm{Wu}$ et al.; ${ }^{31}$ Kikuchi et al. ${ }^{35}$ ). There were only three trials conducted double-blinding trials (Wu et al.; ${ }^{31}$ Seguin et al.; ${ }^{33}$ Zhang et al. $^{36}$ ). The remaining trials did not clearly describe the process of blinding in their studies.

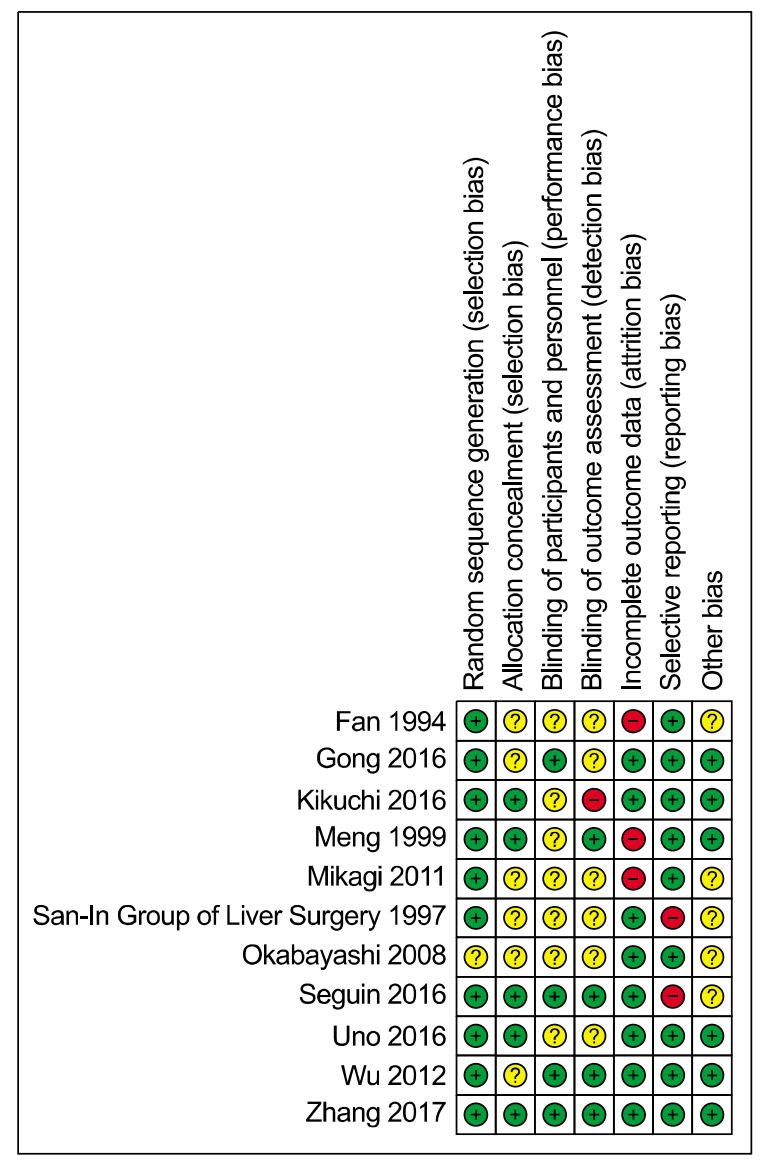

Fig. 4. Risk of bias summary: review authors' judgements about each risk of bias item for each included study.

\section{Incomplete date outcome}

Some of the trials ${ }^{26,28,30}$ (approximately 25\%) were considered to have a high risk of attrition bias because of missing or incomplete dichotomous or continuous out- 
come data.

Selective reporting

Most of the published reports (more than 75\%) have included all expected outcomes so that they can be entered in the meta-analysis. We classified them as having a low risk of selective reporting bias.

Other bias

Of the eleven included studies, five reported dropouts after randomisation (Mikagi et al.; ${ }^{30}$ Seguin et al.; ${ }^{33}$ Uno et al.; ${ }^{34}$ Kikuchi et al.; ${ }^{35}$ Zhang et al. ${ }^{36}$ ), two ${ }^{30,33}$ were re- garded as high risk because the dropout rate was $>25 \%$.

Publication bias

Funnel plots for log RR (mean difference in the case of hospital stay) on vertical Y-axis and effect size on horizontal $\mathrm{X}$-axis from the included studies were used to assess possible publication bias.

Funnel plots as illustrated in the Fig. $5 \mathrm{~A}$ to D showed only minor asymmetry indicates the presence of reporting bias. Visual inspection of the funnel plot indicates that absence of larger studies and exaggerated intervention effect
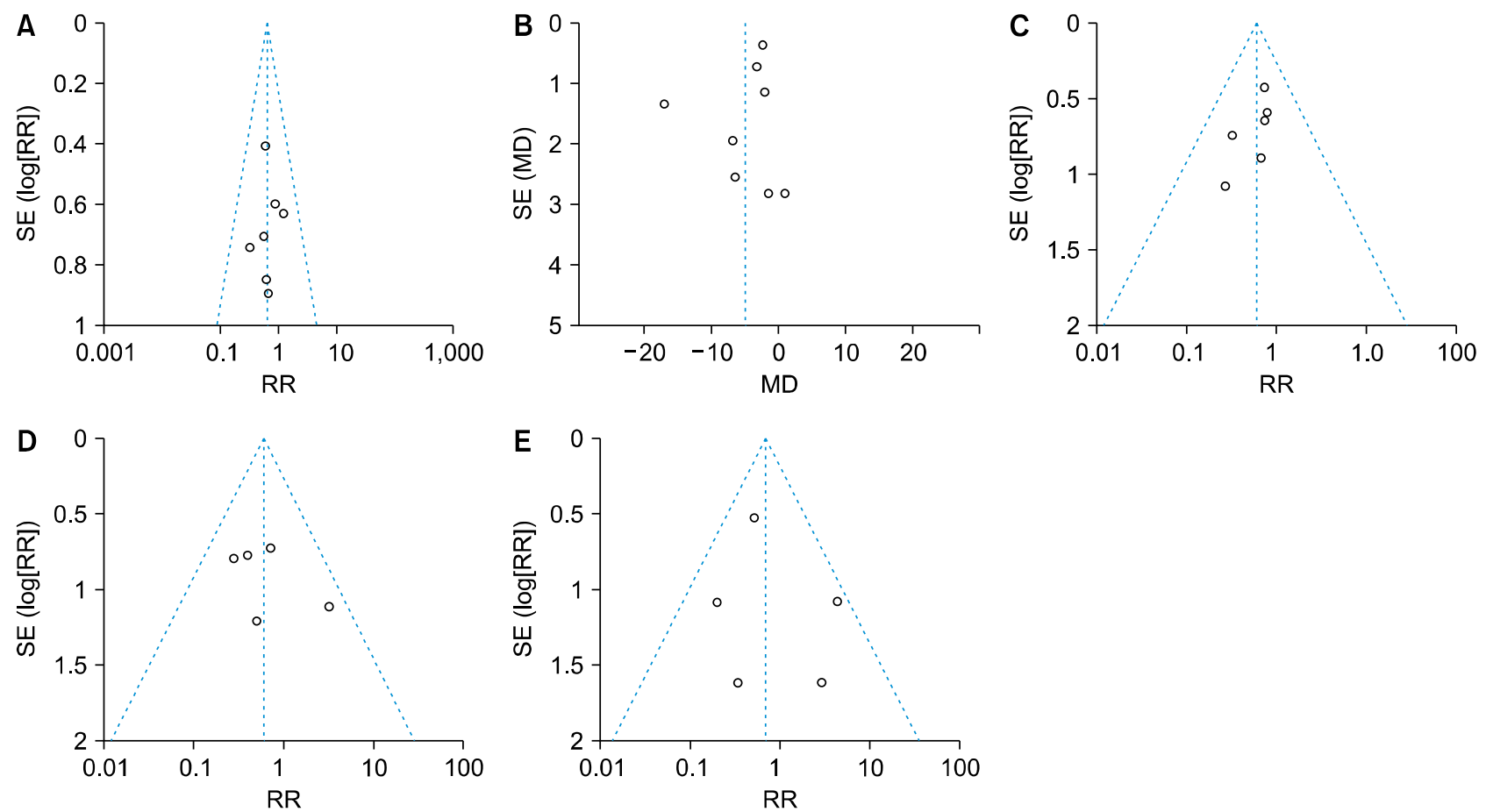

Fig. 5. Test of heterogeneity of selected randomized controlled trials on clinical impact of perioperative immunonutrition in hepatectomy. Funnel plots of comparison: immunonutrition versus controlled group for outcomes based on overall pooled data for (A) wound infection (B) length of hospital stay (C) bile leak (D) liver failure (E) mortality.

\begin{tabular}{|c|c|c|c|c|c|c|c|c|c|c|}
\hline \multirow{3}{*}{$\begin{array}{l}\text { Study or subgroup } \\
\text { Fan } 1994\end{array}$} & \multicolumn{2}{|c|}{ IMN } & \multicolumn{2}{|c|}{ Control } & \multirow[b]{2}{*}{ Weight } & \multirow{2}{*}{$\begin{array}{c}\text { Risk ratio } \\
\mathrm{M}-\mathrm{H}, \text { random, } 95 \% \mathrm{Cl}\end{array}$} & \multirow{2}{*}{\multicolumn{4}{|c|}{$\begin{array}{c}\text { Risk ratio } \\
\mathrm{M}-\mathrm{H} \text {, random, } 95 \% \mathrm{Cl}\end{array}$}} \\
\hline & Events & Total & Events & Total & & & & & & \\
\hline & 3 & 64 & 5 & 60 & $8.4 \%$ & $0.56[0.14,2.25]$ & & $\Longrightarrow$ & - & \\
\hline Gong 2016 & 2 & 59 & 3 & 60 & $5.3 \%$ & $0.68[0.12,3.91]$ & & & & \\
\hline Kikuchi 2016 & 5 & 39 & 4 & 38 & $10.6 \%$ & $1.22[0.35,4.19]$ & & - & & \\
\hline Meng 1999 & 4 & 21 & 5 & 23 & $11.7 \%$ & $0.88[0.27,2.83]$ & & 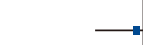 & - & \\
\hline Okabayashi 2008 & 2 & 40 & 11 & 72 & $7.6 \%$ & $0.33[0.08,1.40]$ & &. & & \\
\hline Seguin 2016 & 2 & 18 & 3 & 17 & $5.8 \%$ & $0.63[0.12,3.32]$ & & . & - & \\
\hline Uno 2016 & 6 & 20 & 10 & 20 & $25.2 \%$ & $0.60[0.27,1.34]$ & & $\rightarrow-$ & & \\
\hline Zhang 2017 & 9 & 157 & 15 & 155 & $25.5 \%$ & $0.59[0.27,1.31]$ & & $\rightarrow$ & & \\
\hline Total $(95 \% \mathrm{Cl})$ & & 418 & & 445 & $100.0 \%$ & $0.65[0.43,0.96]$ & & $\Delta$ & & \\
\hline Total events & 33 & & 56 & & & & & & & \\
\hline $\begin{array}{l}\text { Heterogeneity: Tau } \\
\text { Test for overall effe }\end{array}$ & $\begin{array}{l}.00 ; \mathrm{Chi}^{2} \\
\mathrm{Z}=2.13(\end{array}$ & $\begin{array}{l}=2.24, \\
=0.03)\end{array}$ & $7(p=0$. & $5) ; 1^{2}=($ & & & 0.001 & $\begin{array}{l}0.1 \\
\text { IMN }\end{array}$ & $\begin{array}{cc}10 \\
\text { Control }\end{array}$ & 1,000 \\
\hline
\end{tabular}

Fig. 6. Comparison IMN Vs control group. Outcome: wound infection. 
estimate (RR) produced by smaller studies may contribute to the publication bias. There was no asymmetry for mortality (Fig. 5E).

\section{Quantitative data}

Primary outcome

(a) Wound infection (or surgical site infection)

Eight trials ${ }^{26,28,29,32-36}$ reported wound infection rate following hepatectomy. The infectious complication rate was $7.89 \%$ (33/418) in IMN group, while $12.58 \%(56 / 445)$ in the control group. There was statistically significant effect

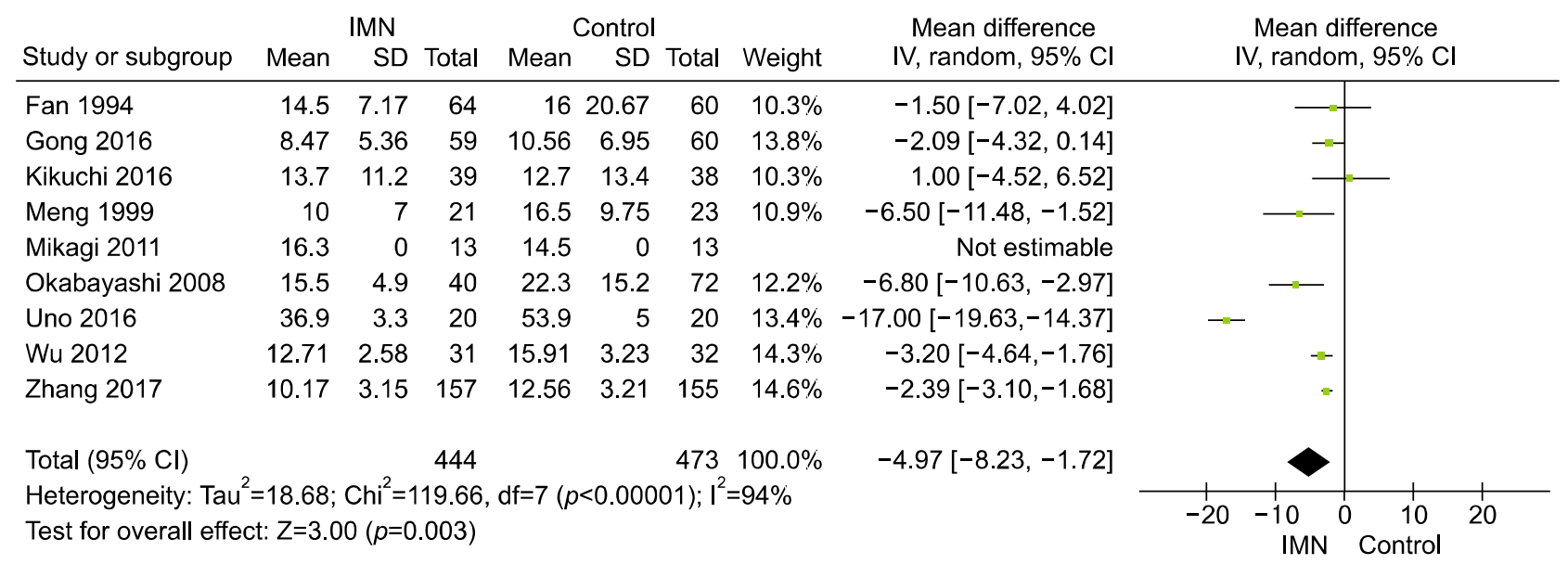

Fig. 7. Comparison IMN Vs control group. Outcome: LOS.

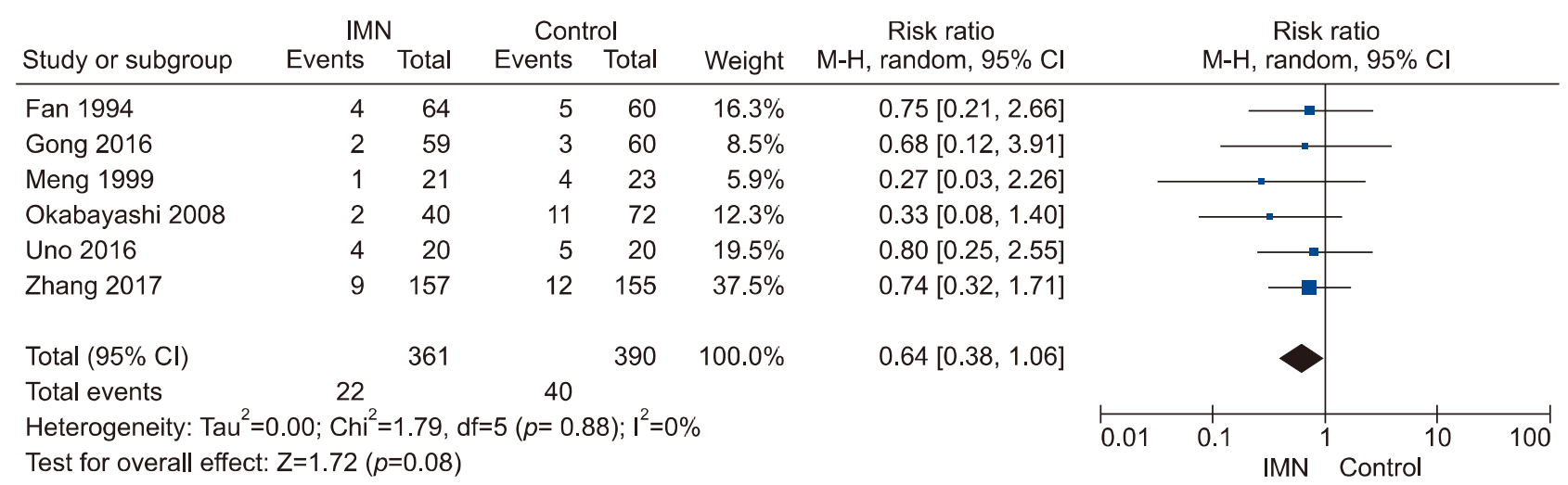

Fig. 8. Comparison IMN Vs control group. Outcome: bile leak.

\begin{tabular}{|c|c|c|c|c|c|c|c|c|c|c|}
\hline \multirow{3}{*}{$\begin{array}{l}\text { Study or subgroup } \\
\text { Gong } 2016\end{array}$} & \multicolumn{2}{|c|}{ IMN } & \multicolumn{2}{|c|}{ Control } & \multirow{2}{*}{ Weight } & \multirow{2}{*}{$\begin{array}{c}\text { Risk ratio } \\
\mathrm{M}-\mathrm{H} \text {, random, } 95 \% \mathrm{Cl}\end{array}$} & \multirow{2}{*}{\multicolumn{4}{|c|}{$\begin{array}{c}\text { Risk ratio } \\
\mathrm{M}-\mathrm{H}, \text { random, } 95 \% \mathrm{Cl}\end{array}$}} \\
\hline & & & & & & & & & & \\
\hline & 1 & 59 & 2 & 60 & $10.3 \%$ & $0.51[0.05,5.46]$ & & & & \\
\hline Kikuchi 216 & 3 & 39 & 4 & 38 & $28.4 \%$ & $0.73[0.18,3.05]$ & & $\longrightarrow$ & & \\
\hline Meng 1999 & 3 & 21 & 1 & 23 & $12.2 \%$ & $3.29[0.37,29.20]$ & & & $=$ & \\
\hline Uno 2016 & 2 & 20 & 5 & 20 & $25.2 \%$ & $0.40[0.09,1.83]$ & & & - & \\
\hline Zhang 2017 & 2 & 157 & 7 & 155 & $24.0 \%$ & $0.28[0.06,1.34]$ & & & - & \\
\hline Total $(95 \% \mathrm{Cl})$ & & 296 & & 296 & $100.0 \%$ & $0.58[0.27,1.24]$ & & & & \\
\hline Total events & 11 & & 19 & & & & & & & \\
\hline \multicolumn{7}{|c|}{ Heterogeneity: $\mathrm{Tau}^{2}=0.00 ; \mathrm{Chi}^{2}=3.59, \mathrm{df}=4(p=0.46) ; \mathrm{I}^{2}=0 \%$} & \multirow[t]{2}{*}{0.01} & \multirow[t]{2}{*}{0.1} & 110 & 100 \\
\hline Test for overall effe & $Z=1.41$ & $=0.16)$ & & & & & & & Control & \\
\hline
\end{tabular}

Fig. 9. Comparison IMN Vs control group. Outcome: liver failure. 
on the rate of wound infection between the two groups in this outcome (RR $0.65,95 \%$ CI 0.43 to 0.96 ) (Fig. 6). There was no heterogeneity in these included studies $\left(\mathrm{I}^{2}=0 \%\right)$.

\section{(b) Length of stay (LOS)}

A pooled analysis of eight studies ${ }^{26,28-32,34-36}$ revealed length of stay was significantly shorter in IMN group (MD -4.97 days, 95\% CI -8.23 to -1.72 ) (Fig. 7). There was significant heterogeneity as denoted by $\mathrm{I}^{2}$. Note that the degree of freedom $\mathrm{Q} / \mathrm{df}$ is $>1 \quad(119.66 / 7=17.09)$ and the $p$-value is significant (0.00001) indicating hetero- geneity is significant. LOS was expressed as median with range [instead of mean and standard deviation (SD)] in two trials ${ }^{26,28}$ (this would be considered as incomplete continuous outcome data in the bias assessment).

\section{(c) Bile leak}

There was no statistically significant difference for this outcome between the two groups (RR $0.64,95 \%$ CI 0.38 to 1.06) (Fig. 8).

\section{(d) Liver failure}

There was no statistically significant difference between the two groups in this outcome (RR $0.58,95 \% \mathrm{CI}$

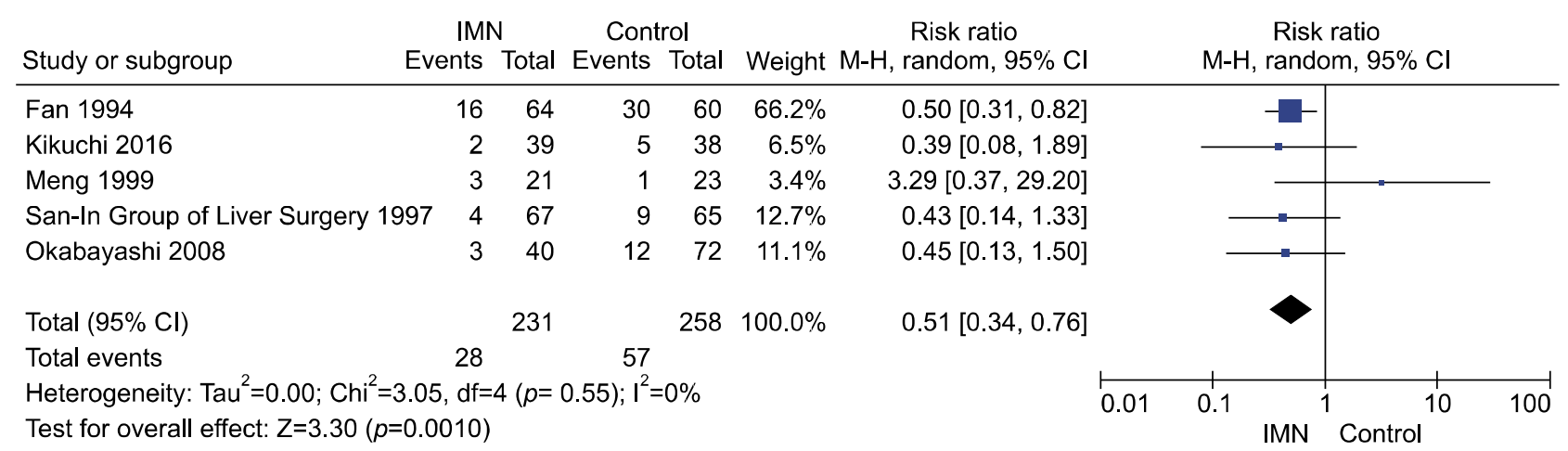

Fig. 10. Comparison IMN Vs control group. Outcome: ascites.

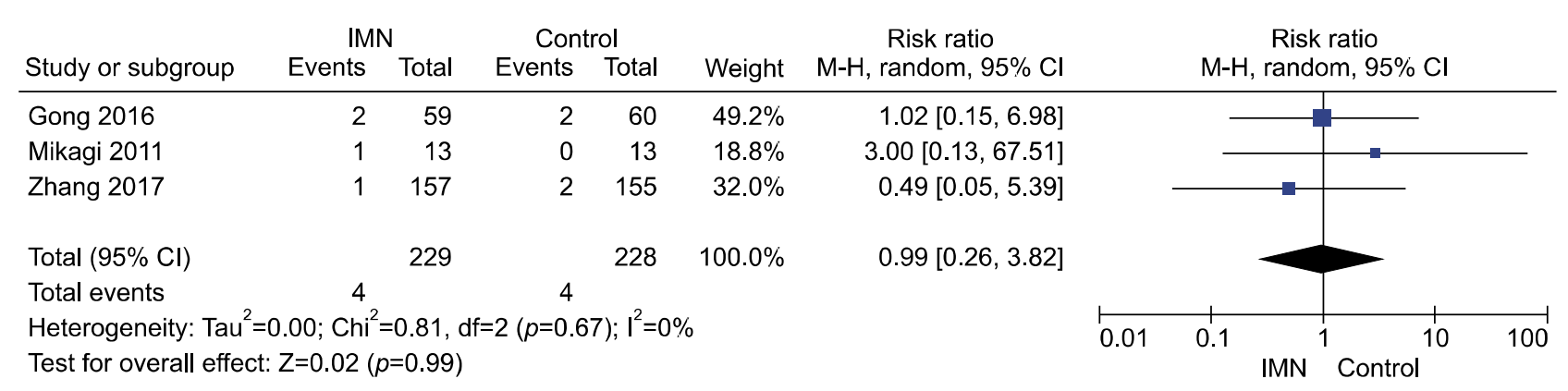

Fig. 11. Comparison IMN Vs control group. Outcome: ileus.

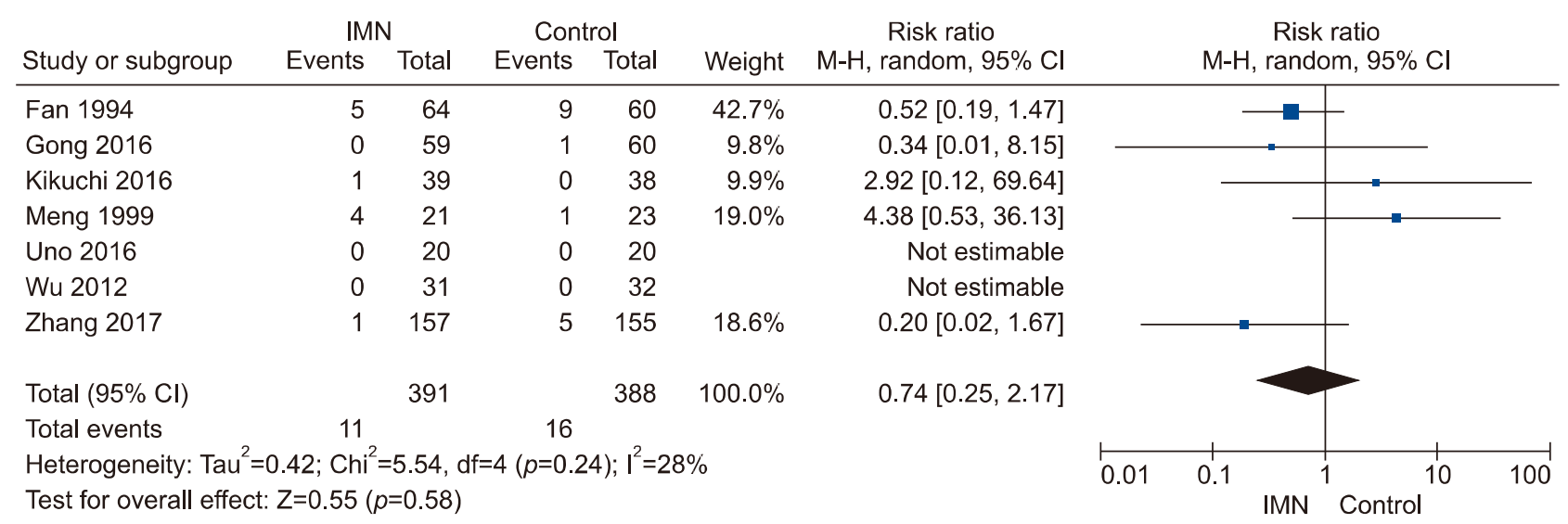

Fig. 12. Comparison IMN Vs control group. Outcome: mortality. 
0.27 to 1.24 ) (Fig. 9).

\section{(e) Ascites}

There was statistically significant difference between the two groups in this outcome (RR $0.51,95 \%$ CI 0.34 to 0.76 ) (Fig. 10). Ascites was significantly reduced in the IMN group.

\section{(f) Ileus}

There was no statistically significant difference between the two groups in this outcome (RR 0.99, 95\% CI 0.26 to 3.82 ) (Fig. 11).

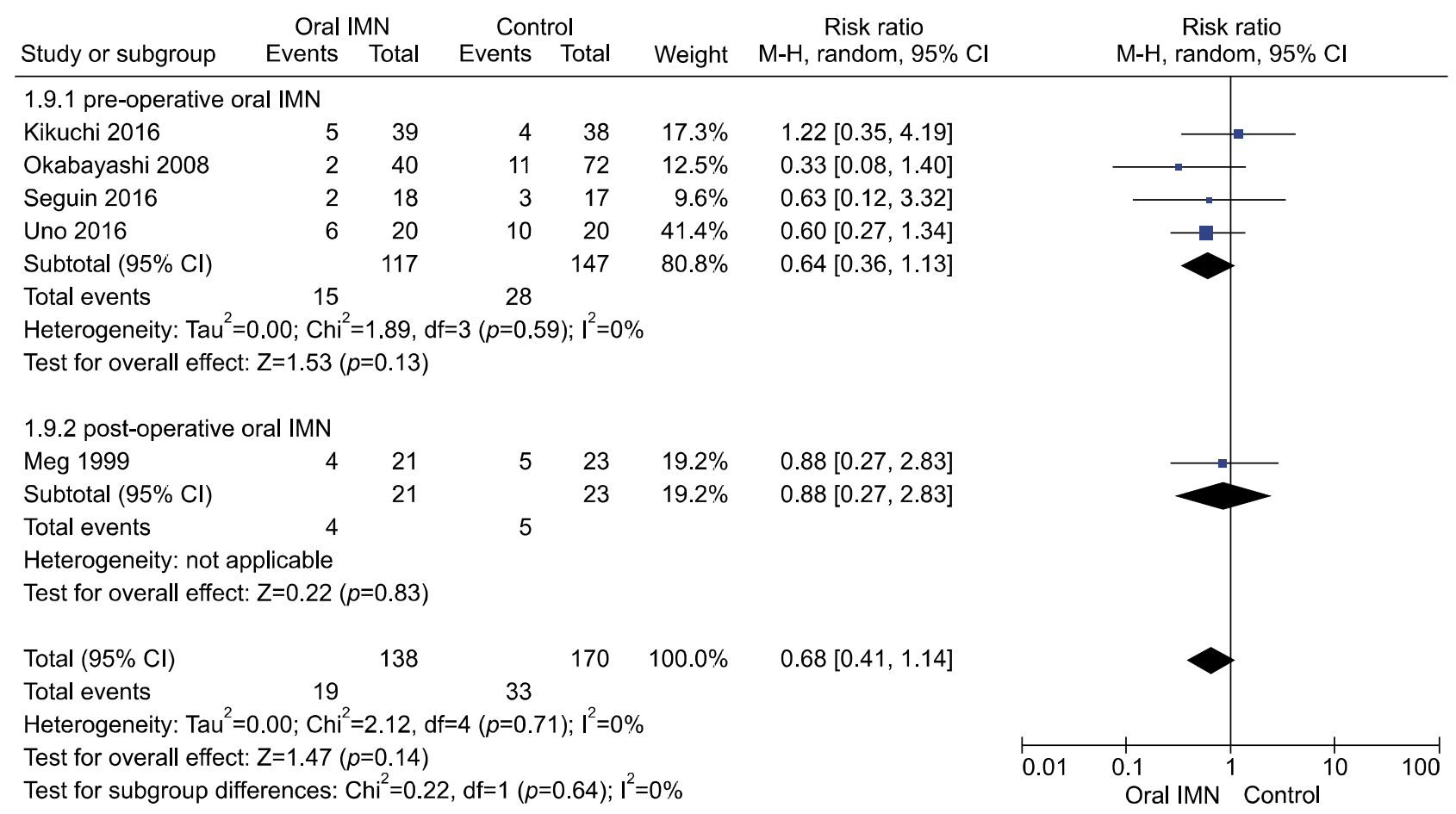

Fig. 13. Effect of preoperative and post-operative oral IMN on wound infection.

\begin{tabular}{|c|c|c|c|c|c|c|c|c|c|c|}
\hline \multirow{2}{*}{ Study or subgroup } & \multicolumn{2}{|c|}{ Parenteral IMN } & \multicolumn{2}{|c|}{ Control } & \multirow[b]{2}{*}{ Weight } & \multirow{2}{*}{$\begin{array}{c}\text { Risk ratio } \\
\mathrm{M}-\mathrm{H}, \text { random, } 95 \% \mathrm{Cl}\end{array}$} & \multirow{2}{*}{\multicolumn{4}{|c|}{$\begin{array}{c}\text { Risk ratio } \\
\mathrm{M}-\mathrm{H}, \text { random, } 95 \% \mathrm{Cl}\end{array}$}} \\
\hline & & & Events & Total & & & & & & \\
\hline \multicolumn{11}{|c|}{ 1.8.1 pre-operative parenteral IMN } \\
\hline Fan 1994 & 3 & 64 & 5 & 6 & $34.2 \%$ & $0.06[0.02,0.18]$ & & & & \\
\hline Subtotal $(95 \% \mathrm{Cl})$ & & 64 & & 6 & $34.2 \%$ & $0.06[0.02,0.18]$ & & & & \\
\hline Total events & 3 & & 5 & & & & & & & \\
\hline \multicolumn{11}{|c|}{ Heterogeneity: not applicable } \\
\hline \multicolumn{11}{|c|}{ Test for overall effect: $Z=4.86(p<0.00001)$} \\
\hline \multicolumn{11}{|c|}{ 1.8.2 post-operative parenteral IMNtrition } \\
\hline Gong 2016 & 2 & 59 & 3 & 60 & $28.5 \%$ & $0.68[0.12,3.91]$ & & & & \\
\hline Zhang 2017 & 9 & 157 & 15 & 155 & $37.3 \%$ & $0.59[0.27,1.31]$ & & & & \\
\hline Subtotal $(95 \% \mathrm{Cl})$ & & 216 & & 215 & $65.8 \%$ & $0.61[0.29,1.25]$ & & & & \\
\hline Total events & 11 & & 18 & & & & & & & \\
\hline \multicolumn{11}{|c|}{ Heterogeneity: $\operatorname{Tau}^{2}=0.00 ; \mathrm{Chi}^{2}=0.02, \mathrm{df}=1(p=0.89) ; \mathrm{I}^{2}=0 \%$} \\
\hline \multicolumn{11}{|c|}{ Test for overall effect: $Z=1.35(p=0.18)$} \\
\hline Total $(95 \% \mathrm{Cl})$ & & 280 & & 221 & $100.0 \%$ & $0.28[0.05,1.53]$ & & & & \\
\hline Total events & 14 & & 23 & & & & & & & \\
\hline \multicolumn{11}{|c|}{ Heterogeneity: $\operatorname{Tau}^{2}=1.88 ; \mathrm{Chi}^{2}=12.54, \mathrm{df}=2(p=0.002) ; I^{2}=84 \%$} \\
\hline \multicolumn{7}{|c|}{ Test for overall effect: $Z=1.48(p=0.14)$} & 0.01 & 0.1 & 110 & 100 \\
\hline
\end{tabular}

Fig. 14. Effect of preoperative and post-operative parenteral IMN on wound infection. 
Secondary outcome

\section{(a) Mortality}

Seven out of eleven RCTs, ${ }^{26,28,31,32,34-36}$ enrolling 391 patients in IMN group and 388 patients in control group, reported postoperative mortality. There was no statistically significant difference between the two groups in this outcome (RR 0.74, 95\% CI 0.25 to 2.17) (Fig. 12).

\section{Subgroup and sensitivity analysis}

Subgroup analysis was performed to evaluate the effect of early administration of IMN and parenteral IMN on primary outcomes of interest (wound infection and length of hospital stay). There was no statistical significant difference of the timing of IMN in oral and parenteral IMN subgroup on wound infection (Figs. 13 and 14). We observed a reduction in the length of hospital stay, which was not statistically significant in the oral IMN subgroup (MD -7.54 days, 95\% CI -15.42 to 0.33 ) (Fig. 15) but was statistically significant in the parenteral IMN subgroup (MD -2.50 days, $95 \% \mathrm{CI}-3.11$ to -1.89 ) (Fig.

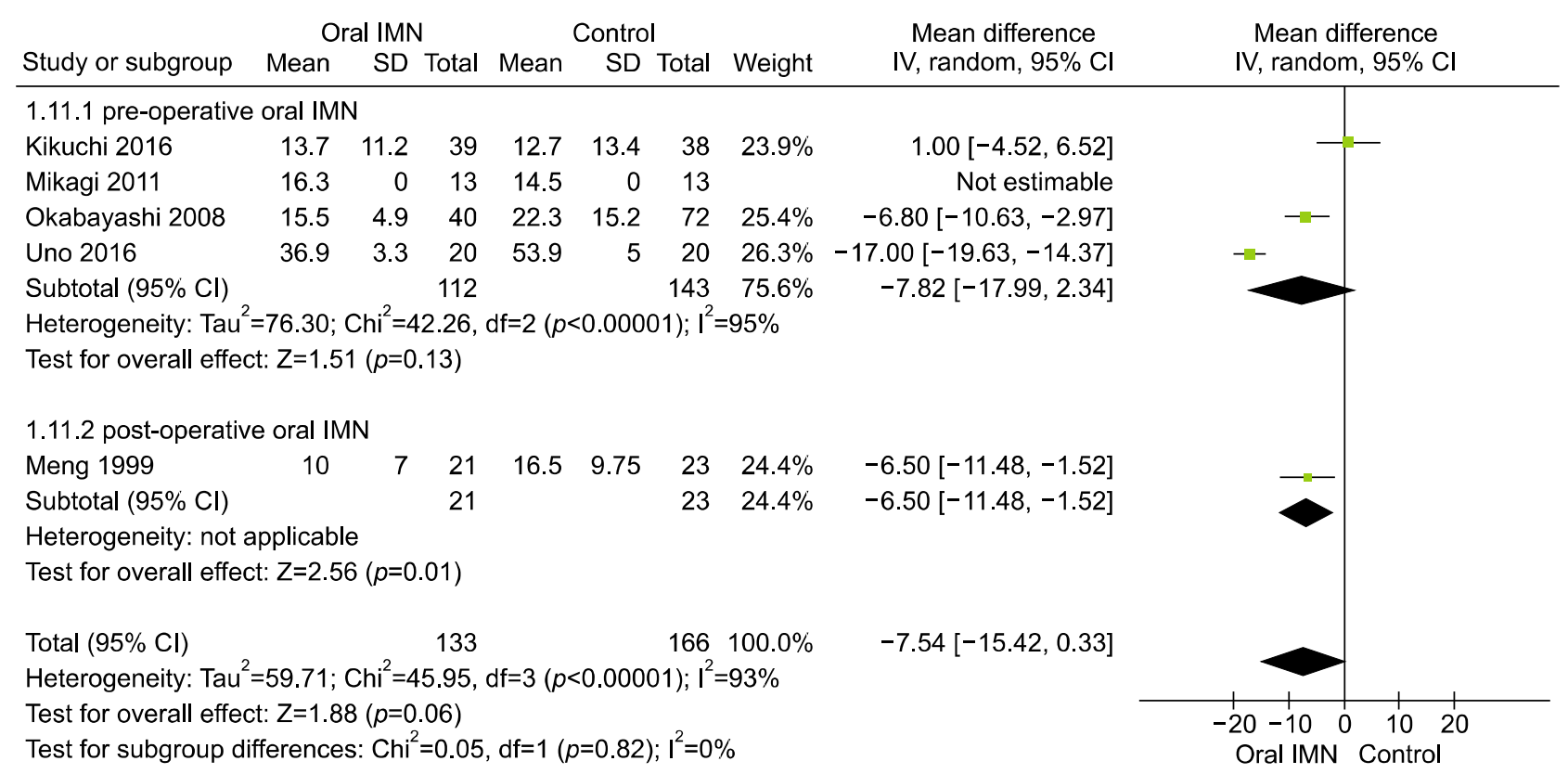

Fig. 15. Effect of preoperative and post-operative oral IMN on LOS.

\begin{tabular}{|c|c|c|c|c|c|c|c|c|c|c|c|c|}
\hline \multirow{2}{*}{ Study or subgroup } & \multicolumn{3}{|c|}{ Parenteral IMN } & \multicolumn{3}{|c|}{ Control } & \multirow{2}{*}{ Weight } & \multirow{2}{*}{$\begin{array}{c}\text { Mean difference } \\
\text { IV, random, } 95 \% \mathrm{Cl}\end{array}$} & \multirow{2}{*}{\multicolumn{4}{|c|}{$\begin{array}{c}\text { Mean difference } \\
\text { IV, random, 95\% Cl }\end{array}$}} \\
\hline & & & Total & Mean & & Total & & & & & & \\
\hline \multicolumn{13}{|c|}{ 1.10.1 pre-operative parenteral IMN } \\
\hline Fan 1994 & 14.5 & 7.17 & 64 & 16 & 20.67 & 60 & $1.2 \%$ & $-1.50[-7.02,4.02]$ & & & & \\
\hline Subtotal $(95 \% \mathrm{Cl})$ & & & 64 & & & 60 & $1.2 \%$ & $-1.50[-7.02,4.02]$ & & & & \\
\hline \multicolumn{13}{|c|}{ Heterogeneity: not applicable } \\
\hline \multicolumn{13}{|c|}{ Test for overall effect: $Z=0.53(p=0.59)$} \\
\hline \multicolumn{13}{|c|}{ 1.10.2 post-operative parenteral IMN } \\
\hline Gong 2016 & 8.47 & 5.36 & 59 & 10.56 & 6.95 & 60 & $7.4 \%$ & $-2.09[-4.32,0.14]$ & & & & \\
\hline Wu 2012 & 12.71 & 2.58 & 31 & 15.91 & 3.23 & 32 & $17.7 \%$ & $-3.20[-4.64,-1.76]$ & & - - & & \\
\hline Zhang 2017 & 10.17 & 3.15 & 127 & 12.56 & 3.21 & 155 & $73.7 \%$ & $-2.39[-3.10,-1.68]$ & & : & & \\
\hline Subtotal $(95 \% \mathrm{Cl})$ & & & 247 & & & 247 & $98.8 \%$ & $-2.51[-3.12,-1.90]$ & & & & \\
\hline \multicolumn{13}{|c|}{ Heterogeneity: $\mathrm{Tau}^{2}=0.00: \mathrm{Chi}^{2}=1.13, \mathrm{df}=2(p=0.57) ; \mathrm{I}^{2}=0 \%$} \\
\hline \multicolumn{13}{|c|}{ Test for overall effect: $Z=8.08(p<0.00001)$} \\
\hline Total $(95 \% \mathrm{Cl})$ & & & 311 & & & 307 & $100.0 \%$ & $-2.50[-3.11,-1.89]$ & & & & \\
\hline \multicolumn{13}{|c|}{ Heterogeneity: $\mathrm{Tau}^{2}=0.00 ; \mathrm{Chi}^{2}=1.26, \mathrm{df}=3(p=0.74) ; I^{2}=0 \%$} \\
\hline \multicolumn{8}{|c|}{ Test for overall effect: $Z=8.09(p<0.00001)$} & & -10 & -5 & $0 \quad 5$ & 10 \\
\hline \multicolumn{9}{|c|}{ Test for subgroup differences: $\mathrm{Chi}^{2}=0.13, \mathrm{df}=1(p=0.72) ; \mathrm{I}^{2}=0 \%$} & Pare & iteral IMN & $\checkmark$ Control & \\
\hline
\end{tabular}

Fig. 16. Effect of preoperative and post-operative parenteral IMN on LOS. 


\begin{tabular}{|c|c|c|c|c|c|c|c|c|c|c|c|}
\hline Study or subgroup & \multicolumn{2}{|c|}{ IMN } & \multicolumn{2}{|c|}{ Control } & \multicolumn{3}{|c|}{ Risk ratio } & \multicolumn{4}{|c|}{$\begin{array}{c}\text { Risk ratio } \\
\mathrm{M}-\mathrm{H}, \text { random, } 95 \% \mathrm{Cl}\end{array}$} \\
\hline Kikuchi 2016 & 5 & 39 & 4 & 38 & $13.4 \%$ & $1.22[0.35,4.19]$ & Low risk & & $\longrightarrow$ & - & \\
\hline Meng 1999 & 4 & 21 & 5 & 23 & $14.9 \%$ & $0.88[0.27,2.83]$ & Low risk & & $\rightarrow$ & - & \\
\hline Uno 2016 & 6 & 20 & 10 & 20 & $32.0 \%$ & $0.60[0.27,1.34]$ & Low risk & & $\rightarrow$ & & \\
\hline Zhang 2017 & 9 & 157 & 15 & 155 & $32.3 \%$ & $0.59[0.27,1.31]$ & Low risk & & $\rightarrow-$ & & \\
\hline Seguin 2016 & 2 & 18 & 3 & 17 & $7.4 \%$ & $0.63[0.12,3.32]$ & Low risk & & 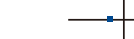 & - & \\
\hline Fan 1994 & 3 & 64 & 5 & 60 & $0.0 \%$ & $0.56[0.14,2.25]$ & Unclear risk & & & & \\
\hline Gong 2016 & 2 & 59 & 3 & 60 & $0.0 \%$ & $0.68[0.12,3.91]$ & Unclear risk & & & & \\
\hline Okabayashi 2008 & 2 & 40 & 11 & 72 & $0.0 \%$ & $0.33[0.08,1.40]$ & Unclear risk & & & & \\
\hline Total $(95 \% \mathrm{Cl})$ & & 255 & & 253 & $100.0 \%$ & $0.70[0.44,1.10]$ & & & & & \\
\hline Total events & 26 & & 37 & & & & & & & & \\
\hline Heterogeneity: Tau & ${ }^{2}=0.00$ & $C h i^{2}=1$ & $.24, d f=4$ & $(p=0$ & .87); $I^{2}=0$ & & & 0.001 & 0.11 & 110 & 1,000 \\
\hline Test for overall effe & $\mathrm{ct}: \mathrm{Z}=1.5$ & $56(p=$ & $0.12)$ & & & & & & IMN & Control & \\
\hline
\end{tabular}

Fig. 17. Sensitivity analysis on primary outcome, wound infection.

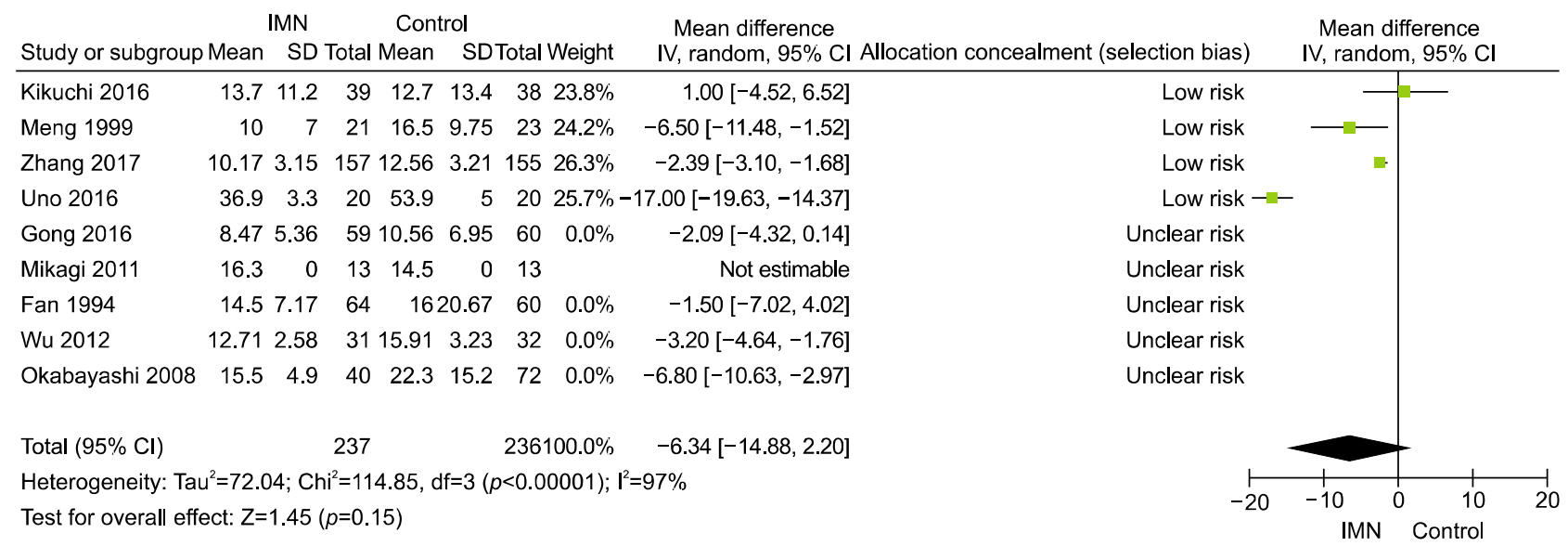

Fig. 18. Sensitivity analysis on primary outcome, LOS.

16) especially if parenteral IMN was administered postoperatively.

On the other hand, sensitivity analysis revealed non-significant difference risk of wound infection (RR 0.70, 95\% CI 0.44 to 1.10 ) (Fig. 17) and length of hospital stay (MD -6.34 days, $95 \%$ CI -14.88 to 2.20 ) (Fig. 18) when trials with 'unclear' or 'high' risk of bias removed. After performing a sensitivity analysis, the heterogeneity still exists in the latter outcome.

\section{DISCUSSION}

Hepatic resection is the gold standard and treatment of choice for primary liver cancers and hepatic metastases. With modern resection techniques, mortality, for even major resection, is now $1-3 \%{ }^{37}$ In well selected patients, 5 -year survival rate after liver resection for primary liver malignancy and for metastatic tumour is $35-38 \%{ }^{38}$ and
$30-40 \%{ }^{39}$ have been reported. Malnutrition is a common problem for patient undergoing liver resection due reduced oral intake, altered metabolic rate, hepatic insufficiency, and the effects of the neoplastic disease itself. Appropriate supplementation with immunonutrition may be beneficial for the patient following major liver resection.

In the context of liver resection or hepatectomy, the role and effectiveness of immunonutrition have not been established. To date, there is no meta-analysis about the effects of peri-operative immunonutrition in patients undergoing elective hepatectomy. The present systematic review and meta-analysis is the first which primarily focused on the effects of enteral immunonutrition in hepatectomy.

Effect of immunonutrition on wound infection. Pooled analysis showed that wound infection rate was significantly reduced in IMN group (RR $0.65,95 \%$ CI 0.43 to 
$0.96 ; \mathrm{X}^{2}=2.24, \mathrm{I}^{2}=0$ per cent, $\left.\mathrm{Z}=2.23 ; p=0.04\right)$. There was no difference of treatment effect in the preoperative and post-operative oral IMN subgroup analysis. Similar result was observed in the parenteral IMN subgroup analysis.

Effect of immunonutrition on length of hospital stay. Although, the combined results showed that IMN had a shorter hospital stay (MD -4.97 days, 95\% CI -8.23 to $-1.72 ; \mathrm{X}^{2}=119.66, \mathrm{I}^{2}=94$ per cent, $\mathrm{Z}=3.00 ; p=0.003$ ), there was significant heterogeneity observed across these studies. Clinical reason for heterogeneity in length of hospital stay may likely be attributed to variable in disease status, local hospital policies or other medical co-morbidities requiring a longer duration of hospitalisation. Similarly, LOS was significantly shorter in the post-operative parenteral IMN subgroup analysis (MD - 2.51 days, 95\% CI -3.12 to -1.90 ) and overall parenteral subgroup analysis (MD -2.50 days, 95\% CI -3.11 to -1.89 ).

Effect of immunonutrition on other post-operative morbidities. There was no statistically significant benefit on other post-operative morbidities of interest [e.g. bile leak (RR 0.64, 95\% CI 0.38 to 1.06), liver failure (RR 0.58, 95\% CI 0.27 to 1.24 ), and ileus (RR 0.99 , 95\% CI 0.26 to 3.82$)$ ]. Interestingly, we found that IMN is associated with a reduced risk of complication related to ascites (RR $0.51,95 \%$ CI 0.34 to 0.76 ).

Effect of immunonutrition on mortality rates. Administration of IMN had no influence on overall pooled mortality rates (RR $0.74,95 \%$ CI 0.25 to $2.17 ; \mathrm{X}^{2}=5.54, \mathrm{I}^{2}=28$ per cent, $\mathrm{Z}=0.55 ; p=0.58$ ). Because of the very low mortality rate in both groups, the present pooled sample size is underpowered to show any differences in mortality rate.

In the past decades, conception of immnonutrition is of increasing interest and has been evaluated by many researchers. ${ }^{8}$ Short courses of preoperative immune-modulating formulas, using combinations of arginine, $\omega-3$ fatty acids, and other nutrients, have been associated with improved surgical outcomes, but there is no conclusive evidence. ${ }^{6}$ The earliest RCTS conducted by Fan et al. ${ }^{26}$ demonstrated that a trend toward decreasing mortality and lowered complication rates. Similarly, a published RCT few years later by San-In Group of Liver Surgery ${ }^{27}$ found that long-term oral nutritional support with BCAAs improves clinical features particularly in patients with advanced cirrhosis after major hepatic resection. The latest and largest trial published recently by Zhang et al. ${ }^{36}$ con- cluded that $\omega-3$ fatty acid-based parenteral IMN significantly improved postoperative recovery for cirrhotic patient with liver cancer following hepatectomy, with a significant reduction in overall mortality and length of hospital stay.

The aim of perioperative nutritional support is to support liver regeneration and function and to prepare the patient metabolically for the insult of the surgical operation. Patient who may benefit from nutrition support should be appropriate identified pre-operatively as optimal nutritional regime could improve clinical outcome and within other postoperative complication. The key components for the appropriate identification of patient at nutritional risk include dietary history, recent weight loss, body fat, muscle mass, presence of fluid accumulation, and grip strength. $^{40}$

In this meta-analysis of eleven RCTs evaluates the effects of IMN in patients undergoing elective liver resection, two important findings-significant reductions in postoperative wound infection and shortened hospital stay were observed when compared with a control group. Decreased infectious complications may lead to shortened postoperative hospital stay. However, the detailed mechanisms of IMN of which decreased the risk of infectious complications are unclear. It is known that both glutamine and $\omega-3$ FAs can down-regulate pro-inflammatory cytokines production and enhance immunity. Studies have shown that the level of interleukin-6 (a pro-inflammatory cytokine) is significant lower in patients who received oral $\mathrm{IMN}^{34}$ and parenteral IMN. ${ }^{31}$ A lowered levels of C-reactive protein and tumour necrosis factor-alpha (TNF- $\alpha$ ) has also been observed in the patient who received perioperative glutamine ${ }^{41}$ and $\omega-3 \mathrm{FAs}^{31}$ respectively.

Usually, only patients with Child-Pugh A disease are considered eligible for liver resection because postoperative mortality rates are higher for patient with higher Child-Pugh class, approaching $50 \%$ for those with Child-Pugh class $\mathrm{C}$ disease. ${ }^{13}$ One study recorded the outcome up to a year follow-up. ${ }^{27}$ To standardise the dichotomous data of this outcome, a cut-off of a month was applied. Therefore, even though positive results were observed i.e. it seems that ascites were significantly less common in the IMN group, but this needs to be interpreted in the context of clinical due to disease status (variable prognosis in cirrhotic liver based on Child-Pugh 
score), malignancy (e.g. HCC, liver metastases and cholangiocarcinoma), hypoalbuminaemia (low albumin) secondary to malnutrition, and the timing when the ascites outcome was measured postoperatively.

Although most of the trials would demonstrate a common objective-to evaluate the effectiveness of perioperative nutritional support in a form of immunonutrition in patient undergoing hepatic resection-however study protocols and end points seem to be varied. For instance, there is no agreed standard regime of which timing of initiation of IMN, duration and total nutritional or calories intake across all studies. It is difficult to dissect certain information pertaining to premorbid status (well-nourished versus malnourish patient); diseased state (cirrhotic, stage of cancer, and severity of hepatitis) and extent of surgery (minor, partial, segmental and major hepatectomy).

The eleven studies that met the inclusion criteria were of varying quality. The majority of the included studies have achieved adequate generation of allocation sequence and allocation concealment as illustrated in Fig. 3. Selection bias (biased allocation to comparison groups), performance bias (unequal provision of care apart from the treatment under evaluation), detection bias (biased assessment of outcomes), and attrition bias (biased occurrence and handling of protocol deviations and loss to follow up) can affect the internal validity of a clinical trial.

As previously mentioned, when random selection of subjects and random allocation to treatment group in the study population are not equally achieved, this could lead to sampling bias (as subset of selection bias). Less than $50 \%$ of all included studies scored low risk of performance and detection bias. This suggests that most of the participants, personnel, and outcome assessors are aware of the intervention allocation after randomisation at a certain point in time during the trials. Blinding can be impossible in some situations (e.g. patients receiving surgery or intervention). It is possible to have adequate randomisation without adequate blinding-as blinding medical staff to patients' assigned intervention may not always be feasible. In this current review, a majority of high risk of incomplete outcome data and selective reporting bias are related to missing data and incomplete outcome for a meta-analysis or failure to report expected outcome that would be expected for such a study respectively.

There is a risk of publication bias in all systematic reviews. Publication bias occurs when relevant published or unpublished studies are not identified and included in a systematic review or meta-analysis. Publication bias can be visually assessed using a funnel plot or a statistical test (e.g. Egger regression test). The funnel plot is a plot of a measure of study size and effect size plotted on the vertical (Y-axis) and horizontal (X-axis) axises respectively. In the absence of publication bias, plots will be distributed symmetrically. Although, the interpretation of the plots is largely subjective, but it offers a visual senses of the relationship between effect size and precision.

In this review, the risk of publication bias was minimised by searching multiple sources of electronic databases and addition sources for both published and unpublished articles, scanning the reference lists, checking registered trial (i.e. at clinicaltrials.gov), searching the 'grey literature', and translating non-English publication. To minimise this further, two co-authors should assess the quality of the included studies, risk of bias and data extraction independently.

It has been recommended that immunonutrition should be initiated from 7 days prior to 7 days after major oncologic GI surgery (e.g. oesophagus, stomach, pancreas, duodenum, hepatobiliary tree). ${ }^{42,43}$ However, in this metaanalysis, pre-operative IMN supplementation did not significantly improve clinical outcomes. An early (preoperative) IMN was not associated with significant shortened length of hospital stay and reduction of wound infection rate (refer to subgroup analysis).

Decision on administration of nutrition supplementation is often made by a multidisciplinary team involving the surgeons, dieticians or nutritionist. Enteral nutrition should be recommended based on clinical indication and patient's nutritional status. Post-operative surgical complications may not be attributed nutritional support alone but also could be due to other factors (surgeon's skills, patient's premorbid, disease and nutritional status). The primary principle is to enhance recovery and reduction of complications associated with the surgery post-operatively. In this regard, nutritional support with immunonutrition in particularly, could enhance and maximise the benefit for the patients and minimising cost related to hospitalisation.

One might argue that immunonutrition should not be routinely chosen over standard oral formula due to a high cost of immunonutrition, lack of strong data to indicate 
an improvement in mortality, and heterogeneity of individual studies. Although positive conclusions were reached, but considering weighting some of the limitations of the data makes broad recommendation about the use of IMN tentative. What appears clear is that it is not associated with any negative outcomes. It seems fair to deduce that when delivered appropriately, both forms of nutritional support can be expected to improve organ function immune response equally in selected patients. Nutritional support can also minimise the metabolic response to surgery and improve protein synthesis and liver regeneration. Therefore it would be beneficial in selected groups of high risk patients (for example diabetic, immunosuppressed, and malnourished).

The high cost of these new nutritional products could be considered a major drawback for their routine use. However, economic analyses carried out by 'blind' economists on data gathered from randomised clinical trials showed that perioperative immunonutrition led to a substantial saving in healthcare resources consumed. ${ }^{44}$ In fact, the saving due to the reduction in postoperative infectious complications by perioperative immunonutrition could offset the higher cost of the supplemental diet. A cost effective analysis would be helpful in determining this.

The strengths of our study are that it addresses a question that is very relevant to the practice of clinical surgery amongst the general surgeons and may potentially lead to the implementation of future guidelines on the use of immuno-modulating diet in patients undergoing hepatectomy. Although the clinical impact of IMN has been evaluated in many reviews but this is the first systematic review and meta-analysis primarily focused on the effects of enteral immunonutrition in liver surgery. We adhered to the current guideline in conducting a systematic review and used a validated method to assess the quality of all selected studies.

We have attempted to perform a subgroup analysis in view of the fact that there were different surgical procedures (major and minor liver resection) and liver diseases; however this is not possible using current statistical software. Other limitations of our meta-analysis are as follows (a) External validity (The extent to which the results of a trial provide a correct basis for applicability to other circumstances) is limited to only adult patients who underwent elective liver resection or hepatectomy; (b) A small sample size recruited in most of the trials, and this may have an impact on the internal validity (The extent to which systematic error (bias) is minimised in a clinical trial) and might mask true effects of the outcomes; (c) A lack of high methodological quality clinical trials and presence of heterogeneity in outcome data. Trials with adequate generation of allocation sequence, adequate allocation concealment and adequate follow-up were considered of high methodological quality.

Heterogeneity exists when the study pools data from a range of studies from different locations, time periods, and patients' pre-operative demographic and disease profile (extent or staging of condition; medical co-morbidities; nutritional status-both malnourished and well-nourished patients). It is difficult to match these outcomes because of the heterogeneity of study populations and study designs and protocols (including differences in the interventions, timing of initiation of IMN and duration of treatment).

A prospective research requires consideration involving a larger patient group, good control for confounding factors, and stratification method to further characterising patients in both experimental and control groups. Stratification is a method of ensuring an equal distribution of key confounding factors between the two groups of a randomised trial. A better methodological quality of future RCT would be required to reduce or to eliminate the confounding variable. In order to improve the quality of RCTs, future studies should also be conducted and reported according to the Consolidated Standards of Reporting Trials (CONSORT) statement or checklist. ${ }^{45}$

In conclusion, wound infection rate was not significantly different between oral and parenteral IMN group. The length of hospital stay was significantly lower in parenteral IMN group than in oral IMN group. The mortality rates were not affected. Immunonutrition should be recommended routinely as part of the nutritional support in the Enhanced Recovery after Surgery (ERAS) protocol for hepatectomy.

\section{ACKNOWLEDGEMENTS}

This research was undertaken as part of the ChM General Surgery. It was previously accepted for poster presentation at the International Surgical Congress of the 
Association of Surgeons of Great Britain and Ireland (ASGBI) in Liverpool, United Kingdom (May 2018), the World Congress of the International Hepato-PancreatoBiliary Association in Geneva, Switzerland (September 2018), the BASO-ACS Annual Scientific Conference, Glasgow, United Kingdom (November 2018), and the Congress of the Asian-Pacific Hepato-Pancreatico-Biliary Association, Seoul, Korea (September 2019).

\section{ORCID}

Chee Siong Wong: https://orcid.org/0000-0003-4818-5609

Raaj Praseedom: https://orcid.org/0000-0001-9541-6356

Siong-Seng Liau: https://orcid.org/0000-0001-5155-4300

\section{AUTHOR CONTRIBUTIONS}

Conceptualization: CSW, SSL. Data collection: CSW. Formal analysis: CSW. Funding acquisition: None. Methodology: CSW. Project administration: RP, SSL. Visualization: None. Writing - original draft: CSW. Writing - review \& editing: CSW, RP, SSL.

\section{REFERENCES}

1. Kehlet H. Multimodal approach to control postoperative pathophysiology and rehabilitation. Br J Anaesth 1997;78:606-617.

2. Weimann A, Braga M, Carli F, Higashiguchi T, Hübner M, Klek $\mathrm{S}$, et al. ESPEN guideline: clinical nutrition in surgery. Clin Nutr 2017;36:623-650.

3. Braga M, Ljungqvist O, Soeters P, Fearon K, Weimann A, Bozzetti F. ESPEN guidelines on parenteral nutrition: surgery. Clin Nutr 2009;28:378-386.

4. McClave SA, Taylor BE, Martindale RG, Warren MM, Johnson $\mathrm{DR}$, Braunschweig $\mathrm{C}$, et al. Guidelines for the provision and assessment of nutrition support therapy in the adult critically ill patient: Society of Critical Care Medicine (SCCM) and American Society for Parenteral and Enteral Nutrition (A.S.P.E.N.). JPEN J Parenter Enteral Nutr 2016;40:159-211.

5. Brennan MF, Pisters PW, Posner M, Quesada O, Shike M. A prospective randomized trial of total parenteral nutrition after major pancreatic resection for malignancy. Ann Surg 1994;220: 436-441; discussion 441-444.

6. Evans DC, Martindale RG, Kiraly LN, Jones CM. Nutrition optimization prior to surgery. Nutr Clin Pract 2014;29:10-21.

7. Lin E, Goncalves JA, Lowry SF. Efficacy of nutritional pharmacology in surgical patients. Curr Opin Clin Nutr Metab Care 1998;1:41-50.

8. Marik PE, Zaloga GP. Immunonutrition in high-risk surgical patients: a systematic review and analysis of the literature. JPEN J Parenter Enteral Nutr 2010;34:378-386.

9. Hegazi RA, Hustead DS, Evans DC. Preoperative standard oral nutrition supplements vs immunonutrition: results of a systematic review and meta-analysis. J Am Coll Surg 2014;219:1078-1087. 10. Probst P, Ohmann S, Klaiber U, Hüttner FJ, Billeter AT, Ulrich $\mathrm{A}$, et al. Meta-analysis of immunonutrition in major abdominal surgery. Br J Surg 2017;104:1594-1608.

11. Reddy SK, Barbas AS, Turley RS, Steel JL, Tsung A, Marsh JW, et al. A standard definition of major hepatectomy: resection of four or more liver segments. HPB (Oxford) 2011;13:494-502.

12. Fischer JE. Fischer's mastery of surgery. 6th ed. Philadelphia: Wolters Kluwer Health/Lippincott Williams \& Wilkins, 2012.

13. Lillemoe K, Jarnagin W. Master techniques in surgery: hepatobiliary and pancreatic surgery. Philadelphia: Wolters Kluwer, 2012.

14. Garden OJ, Parks RW. Hepatobiliary and pancreatic surgery. 5th ed. Edinburgh: Saunders Elsevier, 2014.

15. Cerantola Y, Hübner M, Grass F, Demartines N, Schäfer M. Immunonutrition in gastrointestinal surgery. Br J Surg 2011;98: 37-48.

16. Drover JW, Dhaliwal R, Weitzel L, Wischmeyer PE, Ochoa JB, Heyland DK. Perioperative use of arginine-supplemented diets: a systematic review of the evidence. J Am Coll Surg 2011;212: 385-399, 399.e1.

17. Marimuthu K, Varadhan KK, Ljungqvist O, Lobo DN. A metaanalysis of the effect of combinations of immune modulating nutrients on outcome in patients undergoing major open gastrointestinal surgery. Ann Surg 2012;255:1060-1068.

18. Moher D, Liberati A, Tetzlaff J, Altman DG. Preferred reporting items for systematic reviews and meta-analyses: the PRISMA statement. PLoS Med 2009;6:e1000097.

19. Hozo SP, Djulbegovic B, Hozo I. Estimating the mean and variance from the median, range, and the size of a sample. BMC Med Res Methodol 2005;5:13.

20. Jadad AR, Moore RA, Carroll D, Jenkinson C, Reynolds DJ, Gavaghan DJ, et al. Assessing the quality of reports of randomized clinical trials: is blinding necessary? Control Clin Trials 1996; 17:1-12.

21. Higgins JP, Altman DG, Gøtzsche PC, Jüni P, Moher D, Oxman $\mathrm{AD}$, et al. The Cochrane Collaboration's tool for assessing risk of bias in randomised trials. BMJ 2011;343:d5928.

22. Egger M, Smith GD, Altman DG. Systematic reviews in health care: meta-analysis in context. 2nd ed. London: BMJ, 2001.

23. Mantel N, Haenszel W. Statistical aspects of the analysis of data from retrospective studies of disease. J Natl Cancer Inst 1959; 22:719-748.

24. DerSimonian R, Laird N. Meta-analysis in clinical trials. Control Clin Trials 1986;7:177-188.

25. Higgins JP, Thompson SG. Quantifying heterogeneity in a meta-analysis. Stat Med 2002;21:1539-1558.

26. Fan ST, Lo CM, Lai EC, Chu KM, Liu CL, Wong J. Perioperative nutritional support in patients undergoing hepatectomy for hepatocellular carcinoma. N Engl J Med 1994;331: 1547-1552.

27. San-In Group of Liver Surgery. Long-term oral administration of branched chain amino acids after curative resection of hepatocellular carcinoma: a prospective randomized trial. Br J Surg 1997;84:1525-1531.

28. Meng WC, Leung KL, Ho RL, Leung TW, Lau WY. Prospective randomized control study on the effect of branched-chain amino acids in patients with liver resection for hepatocellular carcinoma. Aust N Z J Surg 1999;69:811-815.

29. Okabayashi T, Nishimori I, Sugimoto T, Maeda H, Dabanaka K, Onishi S, et al. Effects of branched-chain amino acids-enriched nutrient support for patients undergoing liver resection for hepatocellular carcinoma. J Gastroenterol Hepatol 2008;23:1869-1873.

30. Mikagi K, Kawahara R, Kinoshita H, Aoyagi S. Effect of pre- 
operative immunonutrition in patients undergoing hepatectomy; a randomized controlled trial. Kurume Med J 2011;58:1-8.

31. Wu Z, Qin J, Pu L. Omega-3 fatty acid improves the clinical outcome of hepatectomized patients with hepatitis B virus (HBV)associated hepatocellular carcinoma. J Biomed Res 2012;26:395399.

32. Gong Y, Liu Z, Liao Y, Mai C, Chen T, Tang H, et al. Effectiveness of $\omega-3$ polyunsaturated fatty acids based lipid emulsions for treatment of patients after hepatectomy: a prospective clinical trial. Nutrients 2016;8:357.

33. Seguin P, Locher C, Boudjema K, Hamon C, Mouchel C, Malledant $\mathrm{Y}$, et al. Effect of a perioperative nutritional supplementation with Oral Impact ${ }^{\mathbb{R}}$ in patients undergoing hepatic surgery for liver cancer: a prospective, placebo-controlled, randomized, double-blind study. Nutr Cancer 2016;68:464-472.

34. Uno H, Furukawa K, Suzuki D, Shimizu H, Ohtsuka M, Kato $\mathrm{A}$, et al. Immunonutrition suppresses acute inflammatory responses through modulation of resolvin E1 in patients undergoing major hepatobiliary resection. Surgery 2016;160:228-236.

35. Kikuchi Y, Hiroshima Y, Matsuo K, Kawaguchi D, Murakami $\mathrm{T}$, Yabushita $\mathrm{Y}$, et al. A randomized clinical trial of preoperative administration of branched-chain amino acids to prevent postoperative ascites in patients with liver resection for hepatocellular carcinoma. Ann Surg Oncol 2016;23:3727-3735.

36. Zhang B, Wei G, Li R, Wang Y, Yu J, Wang R, et al. n-3 fatty acid-based parenteral nutrition improves postoperative recovery for cirrhotic patients with liver cancer: a randomized controlled clinical trial. Clin Nutr 2017;36:1239-1244.
37. Agarwal A, Borley NR, McLatchie GR. Oxford handbook of operative surgery. 3rd ed. Oxford: Oxford University Press, 2017.

38. Cuschieri A, Steele RJC, Moossa AR. Essential surgical practice: higher surgical training in general surgery. 4th ed. London: Arnold, 2002.

39. Garden OJ, Parks RW. Principles and practice of surgery. 7th ed. Edinburgh: Elsevier, 2018.

40. Jensen GL, Bistrian B, Roubenoff R, Heimburger DC. Malnutrition syndromes: a conundrum vs continuum. JPEN J Parenter Enteral Nutr 2009;33:710-716.

41. Richard V, Dahiya D, Kaman L, Raj P, Behera A. Effect of perioperative glutamine administration on C-reactive protein and liver function tests in patients undergoing hepatic resection. Pol Przegl Chir 2014;86:11-16.

42. Weimann A, Braga M, Harsanyi L, Laviano A, Ljungqvist O, Soeters $\mathrm{P}$, et al. ESPEN guidelines on Enteral nutrition: surgery including organ transplantation. Clin Nutr 2006;25:224-244.

43. Consensus recommendations from the US summitt on immuneenhancing enteral therapy. JPEN J Parenter Enteral Nutr 2001; 25(2 Suppl):S61-S63.

44. Braga M, Gianotti L, Vignali A, Schmid A, Nespoli L, Di Carlo V. Hospital resources consumed for surgical morbidity: effects of preoperative arginine and omega- 3 fatty acid supplementation on costs. Nutrition 2005;21:1078-1086.

45. Altman DG, Schulz KF, Moher D, Egger M, Davidoff F, Elbourne D, et al. The revised CONSORT statement for reporting randomized trials: explanation and elaboration. Ann Intern Med 2001;134:663-694. 